\title{
EXPLORING CONNECTIONS BETWEEN SELF-COMPASSION, MINDFULNESS, AND SOCIAL ANXIETY
}

\section{ELISA MAKADI}

Thesis submitted to the University of Ottawa in partial Fulfillment of the requirements for the MA (Ed) Counselling Psychology

Faculty of Education

University of Ottawa

(C) Elisa Makadi, Ottawa, Canada, 2018 


\begin{abstract}
Social anxiety disorder (SAD) is one of the most common anxiety disorders, with lifetime prevalence rates of 3 to $13 \%$ (Statistics Canada, 2015). SAD is characterized by excessive fear of being negatively judged or scrutinized by others in social or performance situations (Cox, Fleet, \& Stein, 2004). Previous research has demonstrated a relationship between SAD and selfcompassion, and SAD and mindfulness. People with SAD show lower levels of self-compassion compared to healthy controls (Werner et al., 2012), and some research (Neff and Germer, 2013) has indicated that self-compassion can be taught as a skill to enhance levels of self-compassion and overall well-being. As well, mindfulness is a related concept that has been linked to SAD; those with SAD tend to see improvement to their symptoms with mindfulness-based interventions (e.g., Koszycki et al., 2016). Self-compassion and mindfulness are related but distinct constructs that are thought to influence a variety of mental health outcomes. However, few studies have evaluated these constructs concurrently in the context of mental health. The current study examined associations between trait mindfulness, self-compassion and clinical variables in 136 patients with at least moderately severe social anxiety disorder (SAD). Bivariate correlations revealed that higher ratings of self-compassion and facets of mindfulness were associated with less severe social anxiety and depressive symptoms and better functioning and self-esteem. Self-compassion emerged as the strongest predictor of SAD outcomes in regression models. Mediation analysis found that the mindfulness facet Describe served as an indirect mediator in the relationship between self-compassion and severity of clinician-rated social anxiety symptoms. Self-compassion emerged as a mediator between each facet of mindfulness and severity of self- and clinician-rated social anxiety symptoms. Overall, findings from this preliminary cross-sectional study suggest that self-compassion may be a robust indicator of SAD outcomes, and that the ability to label one's experience may partially explain the self-compassion-SAD symptom relationship. Implications for further SAD research are discussed.
\end{abstract}




\section{Acknowledgements}

I would like to thank my thesis supervisor, Dr. Diana Koszycki, for her guidance throughout my time at the University of Ottawa. My thesis is a testament to her feedback, encouragement, and continued support. The lessons I have learned along the way are ones I take forward into my career and work life.

I would like to extend my gratitude to my committee members, Dr. David Smith and Dr. David Trumpower. Your feedback and careful reviews have helped my work immensely.

This thesis was funded by the Canadian Institutes of Health Research and the KoszyckiBradwejn Fund for Graduate Studies in Anxiety Disorders. Clinical trials were funded by the Institut de recherche de l'Hôpital Montfort and the Ontario Mental Health Foundation.

I would also like to thank my family and friends for their support and unending encouragement throughout this process. To my counselling friends, the gift of your friendship alone has made this whole journey worthwhile. 


\section{Table of Contents}

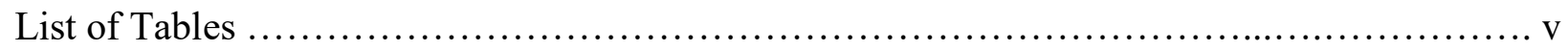

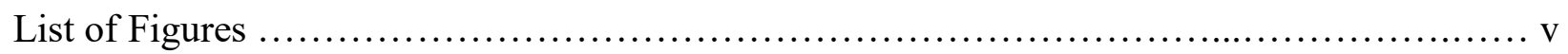

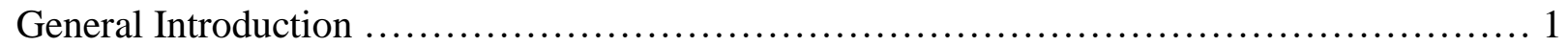

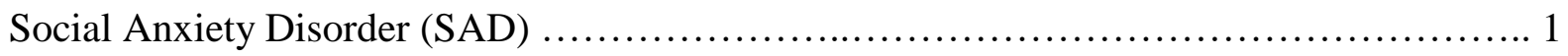

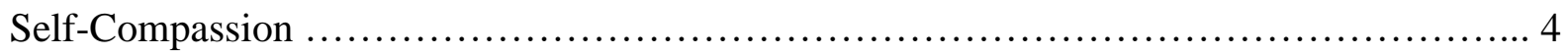

Self-compassion and well-being .............................................. 5

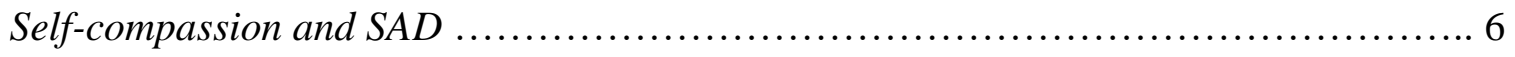

Mindfulness ..................................................................... 7

Mindfulness and well-being .................................................... 8

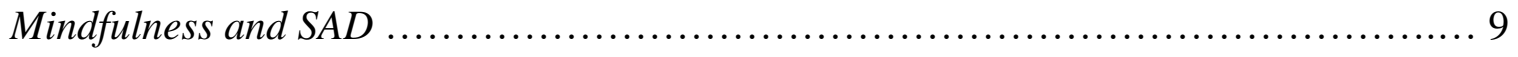

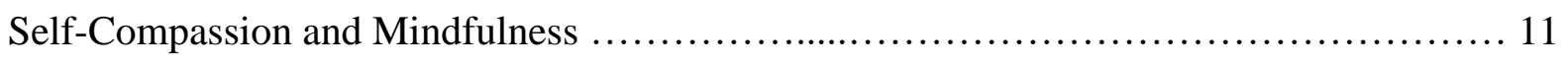

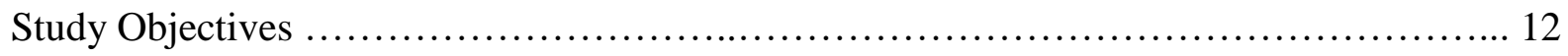

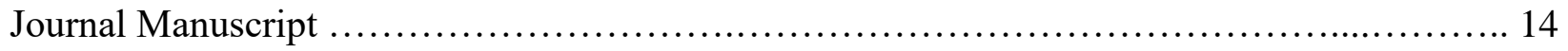

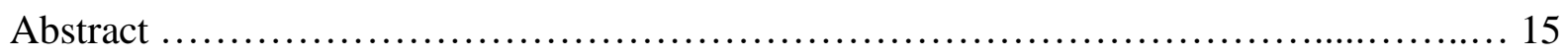

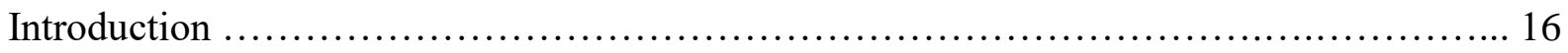

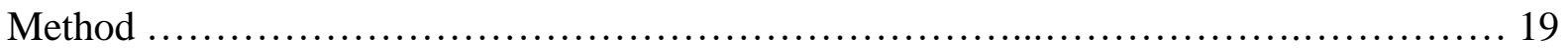

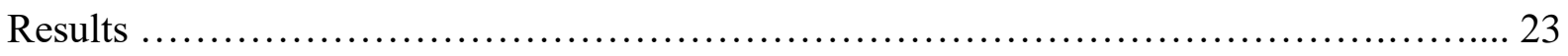

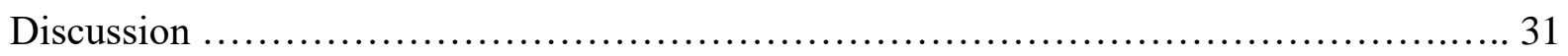

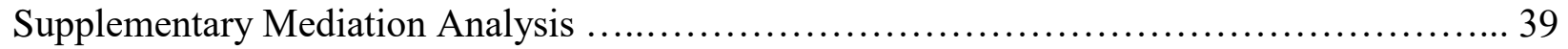

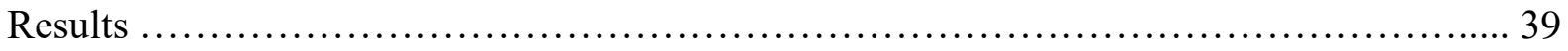

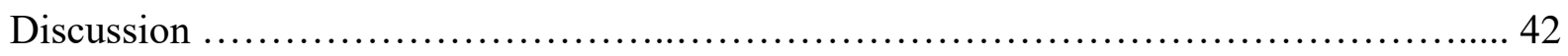

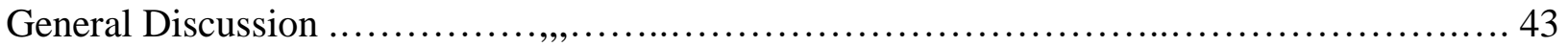

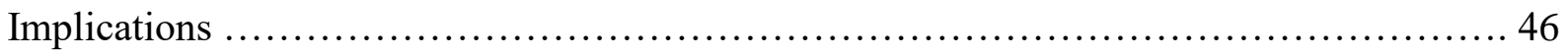

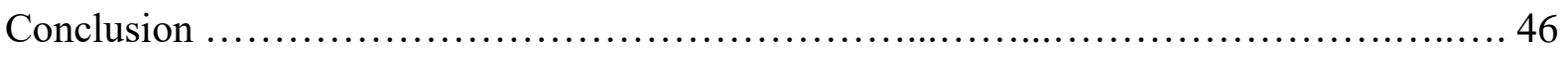

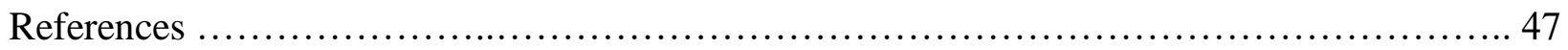

Appendix A: Dominance Analysis Outputs ...................................... 59 


\section{List of Tables}

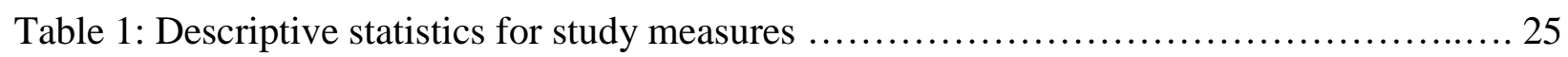

Table 2: Bivariate correlations between mindfulness, self-compassion, and clinical measures . 26

Table 3: Prediction of social anxiety severity based on self-compassion and mindfulness ...... 27

Table 4: Prediction of secondary clinical variables by self-compassion and mindfulness ....... 28

Table 5: Self-compassion as predictor of SAD symptoms (LSAS and SPIN), mediated by

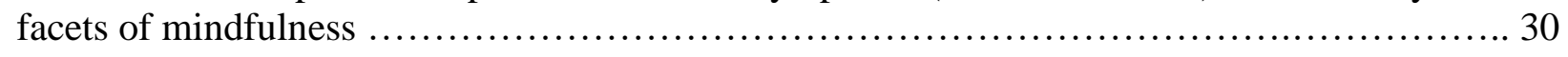

Table 6: Facets of mindfulness as predictors of SAD symptoms (LSAS and SPIN), as mediated by self-compassion ....

\section{List of Figures}

Figure 1a: Mediation between self-compassion and clinician-rated social anxiety severity (LSAS), with the five facets of mindfulness as partial parallel mediators

Figure 1b: Mediation between self-compassion and self-rated social anxiety severity (SPIN), with the five facets of mindfulness as partial parallel mediators

Figure 2: Mediations between each facet of mindfulness and clinician-rated social anxiety severity (LSAS), with self-compassion as a partial mediator

Figure 3: Mediations between each facet of mindfulness and self-rated social anxiety severity (SPIN), with self-compassion as a partial mediator 


\section{General Introduction}

Social anxiety disorder (SAD) is one of the most common anxiety disorders, with lifetime prevalence rates of 3-13\%, a female to male ratio of 3:2, and a mean age of onset around the mid to late ten years (Statistics Canada, 2015; Wittchen \& Fehm, 2001). SAD is characterized by an excessive and persistent fear of being negatively judged or scrutinized by others in social or performance situations and avoidance of feared social situation (American Psychiatric Association, 2013). Those with SAD are fearful of a variety of social situations such as eating or drinking in public, initiating or maintaining a conversation, speaking on the phone, meeting strangers, going to parties, and speaking in front of others (Holt, Heimberg, Hope, \& Liebowitz, 1992). People with SAD are fearful that they will act in a certain way or show anxiety symptoms (e.g., blushing, sweating, trembling) that will result in embarrassment, rejection or humiliation. As a result of this fear, people with SAD either avoid feared social situation or endure them with dread if avoidance is not possible (APA, 2013). SAD differs from shyness by its severity and marked impairments in multiple domains including educational, social, and occupational (Acarturk, Smit, de Graaf, van Straten, ten Have et al., 2009; Acarturk, Smit, de Graaf, van Straten, ten Have et al., 2009; Schneier et al., 1994; Shields, 2004; Stein \& Kean, 2000; Swinson, 2005; Vriends, Becker, Meyer, Michael, \& Margraf, 2007; Wittchen, Fuetsch, Sonntag, Muller, \& Liebowitz, 2000). It has also been linked to comorbid psychological disorders, especially mood disturbances and other anxiety disorders (Acarturk, de Graaf, van Straten, Have, \& Cuijpers, 2008; Acarturk, Smit, de Graaf, van Straten, ten Have et al., 2009; Kessler, 2003; Ruscio et al., 2008; Stein \& Stein, 2008; Wittchen, Kessler, Pfister, \& Lieb, 2000).

The etiological pathways of SAD remain unknown. Etiological components of SAD that have been proposed include genetics, dysregulated neurochemistry, inhibited temperament, environmental influences (e.g., critical parents), behavioural influences (e.g. traumatic conditioning), and cognitive and behavioural factors (Wittchen \& Fehm, 2001). Cognitive and behavior perspectives of SAD emphasize the role of cognitive processes, cognitive content, and behavior in the maintenance of SAD (Heimberg \& Barlow; 1991; Clark and Wells, 1995). Each of these three components (i.e., cognitive processes, cognitive content, and behaviours) contribute to the maintenance of SAD, and are thus important to address in treating the disorder.

Cognitive processes as seen in SAD involve biases in processing social cues and performance-related deficit, as well and anticipatory and post-event processing. Contributing to 
the cognitive patterns associated with SAD is the tendency to ruminate before, during and after a social situation (Clark \& Wells, 1995). This involves processing interactions in detail and with a negative self-view (e.g., pointing out perceived failures, missteps, inadequacies). Clark and Wells (1995) suggest that this post-event rumination is detailed and related to feelings of anxiety that it is strongly encoded in memory. Linked with distorted assumptions and rumination is the tendency to be hypervigilant. Bogels and Mansell (2004) have suggested that those with SAD tend to scan their environment for signs of social threat and have difficulty disengaging from a potential threat. Along with hypervigilance, individuals with SAD report thinking that most people evaluate themselves and others in the same critical, negative ways (Alden, Bieling, \& Wallace, 1994). This biased social perception involves overattending to possible indicators of negative evaluation in others, like frowning or signs of boredom (Rapee \& Heimberg, 1997). By allocating attentional resources to these perceived threat-related social cues, individuals with SAD form negative predictions of what he/she expects to happen in a given social situation. This faulty perception of social cues like frowning in others is compounded by a related cognitive distortion of performance-related deficit; those with SAD often underestimate their own performance in social situations (e.g., Rapee \& Lim, 1992). These cognitive processes contribute to an overall negative, threatening perception of a given social interaction both before and after. Negative predictions elicit more anxiety before a given interaction and continue the cycle of post-even processing after the social interaction (Rapee \& Heimberg, 1997). The cognitive processes that underpin the maintenance of SAD are also connected to cognitive content also involved in SAD symptomology.

Cognitive content includes negative expectations, dysfunctional assumptions and selfbeliefs, and negative schemas. Disordered self-beliefs with regards to social situations are a notable aspect of SAD. Clark and Wells (1995) have theorized that those with SAD have developed dysfunctional negative assumptions about the self (e.g., "I'm stupid"). These faulty thoughts are reinforced over time through social interactions where individuals selectively attend to these assumptions (e.g., "I just said something silly, so obviously I'm stupid. Now there's even more evidence.”). Self-focused attention also contributes to the specific cognitive patterns that reinforce symptoms of SAD. Individuals with SAD tend to attend to internal aspects (e.g., arousal, appearance) in anxiety-provoking social situations, which in turn, results in further negative self-evaluation (Clark \& Wells, 1995). They tend to self-evaluate more critically, 
noticing these internal aspects and forming patterns of negative self-belief like "I'm stupid" (Cox et al., 2004). Beck and Emery (1985) hypothesize that these negative assumptions, thought patterns and dysfunctional assumptions form dysfunctional schemas (i.e., organization of cognitive structures) categorized by hypersensitivity to negative social cues and hyposensitivity to safety cues (see Hope, Rapee, Heimberg, \& Dombeck, 1990). Negative schemas facilitate the continued processing of threatening stimuli, which reinforces the anxious response of the individual with SAD.

Behaviour-related maintenance of SAD involves avoidance and in-situation safety behaviours. As previously mentioned, those with SAD tend to experience physiological symptoms of arousal in feared social situation (e.g. blushing, sweating, shakiness). These physiological symptoms in turn promote more anxiety; research has found that those with SAD believe these signs anxiety will be perceived negatively by others (Clark \& Wells, 1995). These physiological symptoms may be sources of self-focused attention for the individual with SAD; they perceive the physiological symptoms as obvious to others and cause for further negative self-evaluation. To cope with these physiological signs of anxiety, those with SAD often use safety behaviours in social situations. Safety behaviours are actions perceived by the individual as a way to prevent a feared outcome in a social situation (Piccirillo, Dryman, \& Heimberg, 2016). These behaviours are an attempt to minimize anxiety and negative self-evaluation, and can be internal (e.g., planning out and practicing a conversation mentally before speaking at a party) or external (e.g., decreased eye contact) in nature. While these safety behaviours may reduce anxiety in the moment, they prevent the individual from gathering evidence that disconfirms their negative self-beliefs and fears (Piccirillo, Dryman, \& Heimberg, 2016). Indeed, one study that compared the utility of different types of safety behaviours in participants with $\mathrm{SAD}$ found that using safety behaviours like rehearsal and "acting" friendly impeded participants' ability to avoid negative predictions for future interactions; the safety behaviours did not help disconfirm their negative beliefs (Leili Plasencia, Alden, \& Taylor, 2011). Another behavioural aspect of SAD is avoidance; it is common for those with SAD to simply avoid anxiety-provoking social situations (Clark \& Wells, 1995). This avoidance reinforces feelings of anxiety and negative self-evaluation, helping to maintain SAD. Adding to this avoidance along is what some researchers have deemed the vigilance-avoidance hypothesis (e.g. Clark \& Wells, 1995) that suggests that hypervigilance is followed by the defense technique of avoidance. This 
combination of hypervigilance and avoidance can result in enhanced processing of threatening stimuli without the opportunity to sit with this stimulus long enough to see it as non-threatening (Bogels \& Mansell, 2004). Understanding the cognitive and behavioural aspects of SAD is integral to treating this disorder. In relation to these aspects, therapeutic concepts like selfcompassion and mindfulness are also important to consider in how best to treat SAD.

\section{Self-Compassion}

While Western psychology has long examined empathy and compassion for others, it is only more recently that it has begun to consider self-compassion (Neff, 2003). In psychological research, self-compassion is defined as compassion turned inward, representing how we respond to ourselves in response to adversity or failure (Neff, 2016). Neff (2016) conceptualizes selfcompassion as having three main components: self-kindness versus self-judgment, a sense of common humanity versus isolation, and mindfulness versus self-identification. These components combine and mutually interact to create a self-compassionate frame of mind (Neff, 2009). Self-kindness entails showing kindness and understanding towards oneself in the face of failure and adversity as opposed to responding to these circumstances with harsh self-criticism, negative judgment, and rumination (Neff \& Dahm, 2014; Neff, 2003). Common humanity entails seeing one's own imperfections and failings from a broader perspective, acknowledging that suffering, failure and inadequacies are part of the collective human experience and worthy of compassion and kindness (Neff \& Dahm, 2014). The third component of self-compassion, mindfulness, involves the capacity to hold painful emotions in awareness without getting caught up in the experience. Neff and Dahm (2014) specify that mindfulness is an important component of self-compassion because we must be willing to look inward and experience potentially painful emotions in order to truly embrace ourselves with compassion. In this sense, mindfulness involves being aware of our sometimes-negative inner life and "sit with it" without avoidance. This component of self-compassion is best understood in relation to its contrast, overidentification. Often with negative emotions, we tend to get caught up in our reactions to them and link our selves to those reactions. Not over-identifying (and thus being mindful) involves recognizing that our negative thoughts and emotions are separate from our selves - we can take a step back and look at them without assuming they mean anything about our true nature (Neff \& Dahm, 2014). Aside from this operationalization of self-compassion, other conceptualizations help ground what self-compassion is and, flowing from these views, how it can help those with 
SAD.

In addition to Neff's operationalization of self-compassion, the term has also been conceptualized in relation to Paul Gilbert's model of the three-part emotional regulation system. This model comprises a threat detection and protection system that functions to notice potential threats quickly and react with emotions like anxiety or anger and propel us to take action or retreat (i.e., fight or flight response; Gilbert, 2009). The second regulation system is a drive system, which motivates and excites us toward resources and rewards (e.g., food, sex, relationships; Gilbert, 2009). The third regulation system, of particular importance to those with $\mathrm{SAD}$, is the soothing system, which promotes positive, calm feelings when not in a state of threat or danger. Gilbert (2009) describes this soothing system as hardwired to regulate the other two systems; it involves not just an absence of threat, but also a contentment associated with not actively seeking resources or rewards. Gilbert theorizes that anxiety can result when these three regulation systems become unbalanced. If the threat protection and/or drive system become overactive and oversensitive, the individual may find it difficult to feel soothed; this problem is common in those with high levels of self-criticism (Gilbert, 2009). Promoting the soothing system and rebalancing the three systems can be done through self-compassion-focused therapy.

\section{Self-Compassion and Well-Being}

Previous research has considered the impact of self-compassion on well-being, with wellbeing involving having lower stress, lower negative affect, a sense of purpose in life, and overall satisfaction with life (Neely, Schallert, Mohammed, Roberts, \& Chen, 2009). Research findings indicate that self-compassion is positively correlated to well-being (Barnard \& Curry, 2011). In a meta-analysis by MacBeth \& Gumbley (2012) that included 20 studies, self-compassion was associated with lower levels of depression and stress (overall $r=-0.54 ; \mathrm{Z}=-34.02 ; p<.0001$ ). This meta-analysis also considered anxiety disorders, though it did not focus on SAD. For anxiety disorders in general, they found moderate to strong effect sizes supporting selfcompassion as related to lower levels of stress and depression ( $r$ range $=-21$ to -75$)$. A study looking at self-compassion in participants who self-reported mixed anxiety and depression found that self-compassion was a good predictor of symptom severity; that is, those who were more depressed and anxious tended to have lower self-compassion (Van Dam, Sheppard, Forsyth, \& Earleywine, 2011).

Studies have considered links between self-compassion and positive psychological 
outcomes. In a study that compared self-compassion, psychological well-being, and personality traits, higher self-compassion was associated with adaptive psychological variables that were important for attitudinal happiness (Hollis-Walker \& Colosimo, 2011). Interestingly, this study also found that self-compassion was connected to mindfulness. A study by Neff and Germer (2013) looked at teaching mindful self-compassion to a sample of adults from a community sample (with $81 \%$ having prior meditation experience; presence of SAD or other psychological disorders was not measured). They found that self-compassion, mindfulness, and well-being all improved from pretreatment to posttreatment, and that these results were still significant 1 year later. This indicates not only that improving self-compassion improves well-being, but also that self-compassion in itself may be a teachable skill. Another study (Breines \& Chen, 2012) randomized participants into either a self-compassion condition or a control condition for a series of experiments looking at how self-compassion affects motivation to improve personal weaknesses, moral transgressions, and test performance. Results indicated that participants in the self-compassion condition reported more motivation to improve themselves (Breines \& Chen, 2012). Another example of self-compassion and positive psychological outcomes is a study by Sbarra, Smith, and Mehl (2012), who considered factors impacting recovery after a marital separation. This study indicated that adopting self-compassion promoted emotional resiliency in the face of a stressful life situation like separation or divorce.

\section{Self-Compassion and SAD}

Preliminary research suggests that that people with SAD show lower self-compassion. A study by Werner and colleagues (Werner et al., 2012) found that participants with SAD had lower levels of self-compassion than healthy controls. These authors also found that low levels of self-compassion were associated with greater fear of positive and negative evaluation, but not with severity of SAD symptoms in general (Werner et al., 2012). A limitation of this study is the relatively small sample size $(n=72$ participants with SAD).

While few studies have evaluated self-compassion in SAD using a specific selfcompassion scale, other studies reflect SAD's connection related concepts like self-criticism and low self-esteem. For example, Cox and colleagues (2004) found that levels of self-criticism were higher in participants with SAD than in those without a psychiatric disorder; moreover, levels were higher in those with SAD when compared to those with other anxiety disorders. Another study (Cox, Walker, Enns, \& Karpinski, 2002) compared changes in levels of self-criticism in 
those with SAD before, during, and after therapeutic treatment. They found that change in selfcriticism was significantly associated with positive outcome; that is, symptoms of SAD lowered as self-criticism also lowered. A recent study by Iancu, Bodner, \& Ben-Zion (2015) looked at how concepts like self-criticism and self-esteem predicted severity of SAD. Results indicated that low self-esteem, low self-efficacy, high self-criticism, and high dependency predicted SAD severity (all $p<.001$ ). Taken together with Werner and colleagues' study evaluating selfcompassion explicitly, research into critical beliefs and treatment of the self in SAD indicate that these self-critical (i.e., lacking self-compassion) beliefs contribute to SAD; targeting these factors in treatment stands to benefit those with SAD.

\section{Mindfulness}

Mindfulness involves a state of being attentive to what is taking place in the present moment; it is thought to be a naturally occurring individual personality difference or a dispositional trait (Brown \& Ryan, 2003). Previous research into mindfulness as a trait has indicated that levels of mindful attention vary naturally from person to person (Baer, 2011). Trait mindfulness has been associated with positive psychological outcomes like more positive affect and satisfaction with life (Brown \& Ryan, 2003), less reactivity to stress (Bullis, Boe, Asnaani, \& Hofmann, 2014; Sirois \& Tosti, 2012), less rumination (Keng, Smoski, \& Robins, 2011), and stronger cognitive skills like visual working memory and cognitive control (Anicha, Ode, Moeller, \& Robinson, 2012; see Keng et al., 2011, for a review of effects of mindfulness on psychological health).The practice of mindfulness is commonly defined as bringing one's attention to what is happening in the present in an accepting, non-judgmental way, thus increasing trait mindfulness (Baer, Smith, Hopkins, Krietemeyer, \& Toney, 2006; Kabat-Zinn, 2003). In traditional Eastern spiritual traditions like Buddhism, mindfulness is encouraged through meditation, with increases in awareness, insight, and compassion likely to result (KabatZinn, 2000). In more recent years, Western psychology has adapted the Eastern tradition of mindfulness meditation practices for use in clinical settings (Allen, Chambers, Knight, \& Melbourne Academic Mindfulness Interest Group, 2006). Several mindfulness-based interventions have emerged, the most well-researched and supported of which are mindfulnessbased stress reduction (MBSR; see Grossman, Neimann, Schmidt, \& Walach, 2004; Khoury, Sharma, Rush, \& Fournier, 2015) and mindfulness-based cognitive therapy (MBCT; see Chiesa $\&$ Serretti, 2011). During mindfulness practice, one becomes an observer of one's own thoughts, 
sensations and emotions, without attachment, aversion or rumination that often leads to suffering (Baer et al., 2006). Enhancing awareness through mindfulness is thought to be the mechanism that regulate emotions and reduce ruminative thinking, a known risk factor for several psychological disorders (e.g., Khoury et al., 2013).

One common conceptualization of trait mindfulness in psychological research consists of five facets: Observe, Describe, Act with Awareness, Nonjudge, and Nonreact. These facets help researchers understand which skills are cultivated through mindfulness and how these skills contribute to improve psychological functioning (Baer et al., 2008). Understanding each facet is integral to then understanding how each contributes to the overall concept of mindfulness. First, the facet of Observe involves noticing internal and external experiences (Baer et al., 2008). Describe involves labeling internal experiences with words. Acting with Awareness involves attending to one's activities of the present moment - in contrast to acting on "automatic pilot" as humans often tend to do. The facet of Nonjudge involves refraining from taking an evaluative stance toward our internal thoughts and feelings. Finally, the facet of Nonreact involves allowing thoughts and feelings to come and go without getting caught up in them (Baer et al., 2008).

\section{Mindfulness and Well-Being}

Research has begun to consider how mindfulness-based interventions affect psychological well-being. Hofmann and colleagues (2010) conducted a meta-analytic review involving 39 studies and found that mindfulness interventions improved symptoms of anxiety (Hedges's $g=0.63$ ) and mood (Hedges's $g=0.59$ ) in participants with diverse conditions including cancer, anxiety disorders, and depression. Interestingly, the effect sizes were larger (Hedges's $g=0.97$ and 0.95 for anxiety and mood symptoms, respectively) in patients with anxiety and mood disorders. Another meta-analysis by Grossman and colleagues (2004) evaluated the impact of mindfulness-based interventions in a wide range of clinical populations (e.g., cancer, heart disease, attention-deficit hyperactivity disorder, depression, stressed nonclinical sample). Results indicated that mindfulness improved both physical (where applicable) and mental well-being (Cohen's $d=.50$ ). Another study examined the effects of mindfulness intervention on medical patients with both physical and psychological diagnoses (Reibel, Greeson, Brainard, \& Rosenweig, 2001); this study found that the intervention significantly improved both medical and psychological symptoms, as well as overall well-being.

Several other studies have demonstrated that mindfulness improves psychological 
distress. A meta-analysis by Khoury and colleagues (2013) involving 209 studies indicated that mindfulness-based therapies were effective, especially for reducing anxiety and depression (Hedge's $g=.55$ ). A meta-analysis focused on effects of mindfulness on anxiety disorders found similar promising results across 19 studies (Vøllestad, Nielsen, \& Nielsen 2012). The results of this meta-analysis revealed significant effect sizes for both within-group pre- to post-treatment symptoms (Hedges' $g$ effect sizes of 1.08 for anxiety symptoms and 0.85 for depressive symptoms) and between-group pre- to -post-treatment symptoms (Hedges' $g$ of 0.83 for anxiety symptoms and 0.72 for depressive symptoms). Other meta-analyses (Bohlmeijer, Prenger, Taal, \& Cuijpers, 2010; Goyal et al., 2013) indicated that mindfulness-based interventions reduced anxiety to a less significant extent (i.e., only small to moderate effect sizes; Cohen's $d=0.47$ for Bohlmeijer et al., 2010; Cohen's $d=0.38$ at 8 weeks and 0.22 at 3-6 months for Goyal et al., 2013). These results likely reflect the quality of study designs (e.g., both controlled and uncontrolled studies were included), lack of statistical power for calculating intervention effects, and the inclusion of clinical as well as non-clinical populations. Also notable is how mindfulness is operationalized in research, often not specifically to evaluate change.

\section{Mindfulness and SAD}

Though research shows promising results for mindfulness as an intervention for a variety of disorders including anxiety disorders, only recently has research begun to consider mindfulness meditation as a potential intervention for SAD. Like self-compassion, mindfulness may prove particularly effective for this population; aspects of mindfulness stand in contrast to many pathological aspects of SAD. A recent study by Parsons, Luebbe, and Clerkin (2017) found that each facet of mindfulness was associated with both trait social anxiety symptoms and responses to a SAD stressor task. Mindfulness-based treatment may help those with SAD on several levels. Mindfulness' focus on the present moment may present a therapeutic alternative to the negative self-talk and pre- and post-event processing that occurs in SAD. Mindfulness practice also emphasizes observing thoughts without judgment, a potentially positive modification to the self-critical, negative, and judgmental beliefs that affect those with SAD. In terms of physiological arousal and stress responses, mindfulness practice promotes more positive stress appraisals. One study (Arche and Craske, 2010) found that levels of trait mindfulness (which can be improved through mindfulness practice) were associated with more positive stress appraisals and lower subjective stress response. For those with SAD, practicing mindfulness can 
help lower physiological symptoms of arousal elicited by social situations, as well as improve how anxious cognitions are processed and interpreted.

Research has indicated promising results for the mindfulness intervention of MBSR as a treatment for SAD. In a study that compared MBSR and cognitive-behavioural group therapy (CBGT), results indicated that both treatments improved mood, functionality and quality of life; however, CBGT led to more improvement in SAD-related symptomology (Koszycki, Benger, Shlik, \& Bradwejn, 2007). A similar study by Goldin and colleagues (2016) compared MBSR and CGBT for SAD and found that both treatments resulted in similar improvements in SAD symptoms. Interestingly, CGBT resulted in a stronger decrease in avoidance-related behaviours than MBSR.

MBSR was also considered in a recent study by Hjeltnes and colleagues (2017), who conducted an open trial of MBSR in post-secondary students with SAD. Their results indicated that the intervention resulted in improvement for most participants (Cohen's $d=.80$; Hjeltnes et al., 2017). Notably, this study found that the largest effect sizes from pre- to post-treatment were found in self-compassion (Cohen's $d=1.49$ ) and mindfulness (Cohen's $d=1.35$ ). Other studies have also found MBSR to be an effective treatment for SAD (Jazaieri et al., 2012).

Some research has also been conducted regarding the effectiveness of other mindfulnessbased interventions like MBCT for the treatment of SAD. An open trial by Kocovski, Fleming, and Rector (2009) involved using mindfulness and acceptance-based group therapy (which includes enhanced mindfulness through MBCT) to treat SAD; results indicated significant improvement in SAD symptoms, as well as depression and rumination. MBCT was also compared to cognitive-behavioural therapy (CBT) as a treatment for SAD in a small pilot study. The results of this study indicate that MBCT was an effective treatment for SAD, lowering symptoms of social phobia up to 12 months' post-treatment; however, CBT was slightly more efficacious (Piet, Hougaard, Hecksher, \& Rosenberg, 2010).

In addition to trial-based studies, a few studies have considered whether brief one-time mindfulness interventions improve SAD-related symptoms. Vassilopoulos (2008) used a brief, one-session mindfulness intervention in socially anxious participants, finding that it helped reduce anxiety and increased positive thinking. Another study found that a brief, one-time mindfulness intervention decreased negative self-focus in socially anxious participants (Vassilopoulos \& Watkins, 2009). Overall, a handful of studies have examined mindfulness in 
terms of a treatment option for SAD, with results indicating it is a promising option (see Norton, Abbott, Norberg, \& Hunt, 2014, which reviews 9 related studies). Notably, most of these studies have measured mindfulness and SAD symptomology without considering the interplay of other factors like self-compassion.

\section{Self-Compassion and Mindfulness}

Important to distinguish is how self-compassion is related to mindfulness, for both concepts are integral to the present research. Self-compassion and mindfulness are distinct but overlapping concepts (Neff \& Dahm, 2014). Both involve awareness and openness toward painful thoughts and experiences to lessen anxiety and emotional reactivity (Neff \& Dahm, 2014). Researchers have correlated measures of self-compassion and mindfulness and found moderate to strong correlations; so, the two concepts seem to be somewhat linked (Birnie, Speca, \& Carlson, 2010; Van Dam, Sheppard, Forsyth, \& Earleywine, 2011). As previously mentioned, one component of self-compassion is mindfulness; it is important to note, though, that the operationalization of mindfulness used in this component of self-compassion is specified as related to negative thoughts and emotions. This definition of mindfulness is much narrower in scope than the more general concept of mindfulness used in psychological research. As well, there are two other concepts that comprise self-compassion: self-kindness and common humanity. These components of self-compassion do not overlap with what defines mindfulness (Neff \& Dahm, 2014). Thus, one may be mindful and self-compassionate, but the two do not always co-occur. It is possible for someone to be mindful and not particularly self-compassionate (in all three component areas). In the same way, it is possible for someone to be selfcompassionate without exhibiting the more general mindfulness concept (Neff \& Dahm, 2014). Longitudinal research by Bergen-Cico and Cheon (2013) suggests that mindfulness is a foundational element of self-compassions and that self-compassion depends on metacognitive skills that are developed during mindfulness training.

It is notable that very few research studies regarding SAD have measured both mindfulness and self-compassion together. Koszycki and colleagues (2016) conducted a recent clinical trial that investigated the efficacy of a 12-week group-based mindfulness intervention adapted for SAD. The intervention improved symptoms of SAD and also improved self-report ratings of self-compassion and aspects of mindfulness (Observe and Aware). Notably, this study did correlational analysis on self-compassion, mindfulness and symptom improvement, with 
findings indicating that increases in self-compassion were associated with lower social anxiety severity ( $r=-0.57$ and $r=-0.53)$ and increases in mindfulness facets of Describe and Aware were associated with lower social anxiety severity ( $r=-0.52$ and $r=-0.52$, respectively). This study did not, however, explore how the two concepts related to symptom severity. As well, this research has yet to explore whether self-compassion and clinical symptoms of SAD were mediated by one or more facets of mindfulness or Vis versa, with self-compassion as a potential mediator in the mindfulness-SAD clinical symptom relationship. Previous studies have considered self-compassion a macro concept (Bergen-Cico and Cheon, 2013), and based on this assessment and the results of Koszycki and colleagues' study (2016), the current study's objectives and design considered mindfulness as a mediator before the other direction of analysis, as outlined below.

\section{Study Objectives}

At present, research has yet to examine which construct (mindfulness or self-compassion) better predicts SAD symptoms. Further, because the two concepts have yet to be compared in relation to $\mathrm{SAD}$, it could be that a facet of mindfulness (other than the limited conceptualization found in the mindfulness component of self-compassion) mediates the relationship between selfcompassion and SAD symptoms. Because research has yet to consider this possible mediator relationship, the other direction (i.e., self-compassion as mediator between facets of mindfulness and SAD and related symptoms) was also considered to check whether both directions reveal similar implications. Research has yet to examine this possible connection between mindfulness and self-compassion in SAD. The purpose of the current study is to investigate whether levels of mindfulness and self-compassion predict SAD clinical characteristics (i.e., severity of SAD symptoms, depression, quality of life and self-esteem), and to determine which of these constructs is a stronger predictor of SAD severity. My specific research questions and hypotheses are:

1) Are mindfulness and self-compassion associated with, and predictive of, the clinical features of SAD? It is hypothesized that facets of trait mindfulness and self-compassion will predict SAD clinical characteristics.

2) Is the relationship between self-compassion and clinical symptoms of SAD mediated by one or more facets of mindfulness (i.e., Observe, Describe, Act with Awareness, Nonjudge, and Nonreact)? It is hypothesized that the mindfulness facets of Observe and 
Aware (as found in Koszycki et al., 2016) will mediate the relation between selfcompassion and SAD clinical symptoms. However, because research is limited in this area, both potential directions will be explored (see third research question below). 3) Is the relationship between mindfulness and clinical symptoms of SAD mediated by self-compassion?

Research questions 1 and 2 are addressed in the research article portion, and research question 3 is addressed as a supplementary mediation analysis. 
Exploring connections between self-compassion, mindfulness, and social anxiety

\author{
Elisa Makadi ${ }^{1}$ \\ Diana Koszycki ${ }^{1,2,3}$
}

University of Ottawa, Ottawa, Ontario, Canada ${ }^{1}$, Institut savoir l'hôpital Montfort, Ottawa, Ontario, Canada $;{ }^{2}$ and University of Ottawa Brain and Mind Research Institute, Ottawa, Ontario, Canada ${ }^{3}$

Submitted for publication; under review

Corresponding author:

Dr. Diana Koszycki

145 Jean-Jacques Lussier, Ottawa, Ontario, K1N 6N5

Email: dkoszyck@uottawa.ca 


\begin{abstract}
Self-compassion and mindfulness are related but distinct constructs that are thought to influence a variety of mental health outcomes. However, few studies have evaluated these constructs concurrently in the context of mental health. The current study examined associations between trait mindfulness, self-compassion and clinical variables in 136 patients with at least moderately severe social anxiety disorder (SAD). Bivariate correlations revealed that higher ratings of selfcompassion and facets of mindfulness were associated with less severe social anxiety and depressive symptoms and better functioning and self-esteem. Self-compassion emerged as the strongest predictor of SAD outcomes in regression models. Mediation analysis found that the mindfulness facet Describe served as an indirect mediator in the relationship between selfcompassion and severity of clinician-rated social anxiety symptoms. Overall, findings from this preliminary cross-sectional study suggest that self-compassion may be a robust indicator of SAD outcomes, and that the ability to label one's experience may partially explain the selfcompassion-SAD symptom relationship. Implications for further SAD research are discussed.
\end{abstract}

Keywords: Social anxiety disorder, self-compassion, mindfulness, mediation analysis 


\section{Introduction}

Social anxiety disorder (SAD) is one of the most common anxiety disorders, characterized by an excessive and persistent fear of being negatively judged or scrutinized by others in social or performance situations and avoidance of feared social situation (American Psychiatric Association, 2013). Recently, there has been interest in the role of self-compassion in SAD. Self-compassion is defined as compassion turned inward, representing how we respond to ourselves in response to adversity or failure (Neff, 2016). Neff (2016) conceptualizes selfcompassion as having three main components: self-kindness versus self-judgment, a sense of common humanity versus isolation, and mindfulness versus self-identification. These components combine and mutually interact to create a self-compassionate frame of mind (Neff, 2009).

In addition to the concept of self-compassion advanced by Neff, the term has also been conceptualized in relation to Gilbert's model of the three-part emotional regulation system (Gilbert, 2009). This model comprises a threat detection and protection system that functions to notice potential threats quickly, trigger emotional reactions like anxiety or anger, and propel us to take action or retreat (i.e., fight or flight response). The second regulation system is a drive system, which motivates and excites us toward resources and rewards (e.g., food, sex, relationships). The third regulation system, of particular importance to $\mathrm{SAD}$, is the soothing system, which promotes positive, calm feelings when not in a state of threat or danger. Gilbert (2009) describes this soothing system as hardwired to regulate the other two systems; it involves not just an absence of threat, but also a contentment associated with not actively seeking resources or rewards. Gilbert theorizes that anxiety can result when these three regulation systems become unbalanced. If the threat protection and/or drive system become overactive and oversensitive, the individual may find it difficult to feel soothed; this problem is common in those with high levels of self-criticism (Gilbert, 2009).

Self-compassion is associated with higher levels of well-being (Barnard \& Curry, 2011) and lower levels of depression and stress (MacBeth \& Gumbley, 2012). Preliminary research suggests that individuals with SAD show lower self-compassion than healthy controls (Werner et al., 2012), and that low levels of self-compassion correlate with greater fear of positive and negative evaluation, but not with severity of SAD symptoms in general (Werner et al., 2012). Other research has shown that lower levels of self-compassion correlate with more fear of social 
interactions in non-clinical and clinical samples; higher scores on trait post-event processing, a cognitive process that is characterized by negative and prolonged rumination after an anxietyevoking or embarrassing social event; and associated with the maintenance of SAD (Blackie \& Kocovski, 2017). While few studies have evaluated self-compassion in SAD using a specific self-compassion scale, other studies reflect SAD's connection to related concepts like selfcriticism and low self-esteem. For example, participants with SAD are more self-critical than psychiatrically healthy controls and those with other anxiety disorders (Cox, Fleet, \& Stein, 2004), and low self-esteem and high self-criticism predict SAD severity (Iancu, Bodner \& BenZion, 2015). Further, following treatment, symptoms of SAD improve as self-criticism decreases (Cox, Walker, Enns, \& Karpinski, 2002). Taken together with work evaluating self-compassion explicitly, research into critical beliefs and treatment of the self have indicated that these selfcritical (i.e., lacking self-compassion) beliefs contribute to SAD; targeting these factors in treatment stands to benefit those with SAD.

In addition to self-compassion, mindfulness is a related concept that shows connections with SAD symptoms (Goldin \& Gross, 2010). Mindfulness involves a state of being attentive to what is taking place in the present moment and is thought to be a naturally occurring dispositional trait (Brown \& Ryan, 2003). One common conceptualization of trait mindfulness in psychological research consists of five facets: Observe, Describe, Act with Awareness, Nonjudge, and Nonreact. These facets help researchers understand which skills are cultivated through mindfulness training and how these skills contribute to improved psychological functioning (Baer et al., 2008). The facet of Observe involves noticing internal and external experiences (Baer et al., 2008). Describe involves labeling internal experiences with words. Acting with Awareness involves attending to one's activities of the present moment — in contrast to acting on "automatic pilot" as humans often tend to do. The facet of Nonjudge involves refraining from taking an evaluative stance toward our internal thoughts and feelings. Finally, the facet of Nonreact involves allowing thoughts and feelings to come and go without getting caught up in them (Baer et al., 2008). High levels of trait mindfulness has been associated with more positive affect and satisfaction with life (Brown \& Ryan, 2003), less reactivity to stress (Bullis, Boe, Asnaani, \& Hofmann, 2014; Sirois \& Tosti, 2012), less rumination (Keng, Smoski, \& Robins, 2011), and stronger cognitive skills like visual working memory and cognitive control (Anicha, Ode, Moeller, \& Robinson, 2012; Keng et al., 2011). 
Important to distinguish is how self-compassion is related to mindfulness. Selfcompassion and mindfulness are distinct but overlapping concepts (Neff \& Dahm, 2014). Both involve awareness and openness toward painful thoughts and experiences to lessen emotional reactivity (Neff \& Dahm, 2014). Moderate to strong correlations have been found between measures of self-compassion and mindfulness (Birnie, Speca, \& Carlson, 2010; Van Dam, Sheppard, Forsyth, \& Earleywine, 2011), so the two concepts seem to be linked. Mindfulness is one component of self-compassion (Neff \& Dahm, 2014), though the operationalization of mindfulness within Neff and Dahm's definition is specified as related to negative thoughts and emotions. This definition of mindfulness is much narrower in scope than the more general concept of mindfulness used in psychological research. As well, there are two other concepts that comprise self-compassion: self-kindness and common humanity. These components of selfcompassion do not overlap with what defines mindfulness (Neff \& Dahm, 2014). Thus, one may be mindful and self-compassionate, but the two do not always co-occur. It is possible for someone to be mindful and not particularly self-compassionate. In the same way, it is possible for someone to be self-compassionate without exhibiting the more general mindfulness concept (Neff \& Dahm, 2014). On the other hand, longitudinal research by Bergen-Cico and Cheon (2013) suggests that mindfulness is a foundational element of self-compassions and that selfcompassion depends on metacognitive skills that are developed during mindfulness training.

A few studies have measured both mindfulness and self-compassion in individuals with SAD. Koszycki and colleagues (2016) investigated the efficacy of a 12-week group-based mindfulness intervention adapted for SAD that included explicit training in self-compassion. The intervention improved symptoms of SAD and also improved self-report ratings of selfcompassion and aspects of mindfulness (Observe and Aware). Further, decreases in severity of SAD symptoms were associated with increases in self-compassion and the mindfulness facets of Describe and Aware. This study did not, however, explore how the two concepts were related to pretreatment symptom severity. To our knowledge, research has yet to explore how selfcompassion and mindfulness relate to baseline clinical characteristics in individuals with SAD. Accordingly, the aim of this study was to investigate associations between mindfulness, selfcompassion and baseline clinical characteristics in SAD and to determine which of these constructs was a stronger predictor of SAD symptom severity and functioning. We also conducted mediation analysis to explore the relationships between self-compassion, mindfulness, 
and severity of SAD symptoms. Though research is limited in this area, based on previous literature (Koszycki et al., 2016; Bergen-Cico \& Cheon, 2013), we tested whether the facets of mindfulness mediated the relationship between self-compassion and SAD symptom severity. It was hypothesized that the mindfulness facets of Observe and Aware would mediate the relation between self-compassion and severity of SAD symptoms.

\section{Method}

\section{Participants}

Participants were individuals who had participated in one of two randomized trials of a mindfulness-based intervention for SAD. To be included in the studies, participants had to meet DSM-5 criteria for SAD based on the Structured Clinical Interview for DSM disorders (SCID-5; First, Williams, Karg, Spitzer, 2015) and obtain a score of 4 or higher on the Clinical Global Impression-Severity (CGI-S; Guy, 1976) and a score of 25 or lower on the Montgomery-Asberg Depression Rating Scale (MADRS; Montgomery \& Asberg, 1979). Participants were excluded if there was a lifetime history of bipolar disorder or psychotic symptoms, a substance-related disorders in the past year, history of suicide attempt in the past five years, history of self-harm behaviour in the past year, a co-existing medical condition that could alter the presentation of SAD (e.g., Parkinson's disease), or if they were currently receiving any other form of psychological counselling or participating in regular meditation or yoga practice. Participants with comorbid depressive and anxiety disorders were included as long as the SAD was the primary diagnosis and presentation. Additionally, use of psychotropic medications was allowed as long as type and dose of medication remained stable for at least six weeks prior to first screening visit.

\section{Procedure}

Participants initially underwent a telephone pre-screen interview with a research assistant who explained the purpose of the clinical trials, confirmed the presence of SAD symptoms, and ruled out obvious exclusion criteria. Potentially eligible participants were then invited for a faceto-face interview with the study investigators. After the study requirements were reviewed and written informed consent obtained, participants were administered the SCID-I to confirm the primary diagnosis of SAD and other eligibility criteria. The SCID-I was administered by doctoral level clinicians and a graduate student in counselling psychology who was supervised by a doctoral level clinician. Participants were then administered clinician-rated scales to assess the 
severity of SAD and depressive symptoms, and participants who were eligible completed a battery of self-report questionnaires at this visit.

\section{Materials}

Liebowitz Social Anxiety Scale (LSAS). The LSAS (Liebowitz, 1987) is a 24-item clinician-rated measure that assesses fear and avoidance of social performance and interaction situations. Fear is rated on a four-point scale $(0=$ none, $1=$ mild, $2=$ moderate, $3=$ severe $)$ and avoidance is rated on a four-point scale $(0=$ never, $1=$ occasionally, $2=$ often, $3=$ severe $)$. Scores range from 0 to 144, with scores 55-65 indicating moderate social phobia, 65-80 marked social phobia, 80-95 severe social phobia, and scores $>95$ very severe social phobia. The scale has been widely used in treatment outcome studies of SAD and is sensitive to treatment change. The LSAS has good internal consistency (Cronbach's $\alpha=.96$, Heimberg et al., 1999), reliability (Liebowitz, 1987), convergent and discriminant validity (Fresco et al., 2001; Heimberg et al., 1999).

Social Phobia Inventory (SPIN). The SPIN (Connor et al., 2000) is a 17-item self-rated scale that assesses each of the symptom domains of SAD (i.e., fear, avoidance, and physiologic arousal), rated over the past week. Resulting scores range in symptom severity from $<20$ (none), 21-30 (mild), 31-40 (moderate), 41-50 (severe), to > 51 (very severe). The SPIN demonstrates solid psychometric properties, including good test-retest reliability (.89 and .78), internal consistency (Cronbach's $\alpha$ was $0.87-0.94$ ), and convergent and divergent validity, and it is sensitive to treatment change (Connor et al., 2000).

Beck Depression Inventory (BDI-II). The BDI-II (Beck, Steer, \& Brown, 1996) is a 21item self-report measure of severity of depressive symptoms over a 2 -week period. The scale is widely used in treatment studies to monitor the effects of treatment. A total score of 0-13 is considered minimal range, 14-19 is mild, 20-28 is moderate, and 29-63 is severe. The scale has been shown to have good psychometric properties including high internal consistency (e.g., Dozois et al., 1998), high convergent validity, and test-reset reliability (.93 for Beck, Steer, \& Brown, 1996; .96 for Sprinkle et al., 2002).

\section{Social Adjustment Scale - Self-Report (SAS-SR). The SAS-SR (Weissman \&} Bothwell, 1976) is a 42-item self-report measure used to assess social adjustment over the previous two weeks. The scale includes questions in areas addressing role performance, interpersonal relationships, friction, feelings and satisfaction in work, and social and leisure 
activities. The resulting mean score ranges between 1 (normal functioning) and 5 (severe maladjustment). The measure has demonstrated good psychometric properties including internal consistency (Cronbach's $\alpha=.74$, Edwards, Yarvis, Mueller, Zingale, \& Wagman, 1978) and testretest reliability (e.g., Edwards et al., 1978). The scale also has high convergent validity (Weissman \& Bothwell, 1976; Weissman, Olfson, Gameroff, Feder, \& Fuentes, 2001).

Rosenberg Self-Esteem Scale (RSES). The RSES (Rosenberg, 1965) is a 10-item measure of self-esteem that includes five positive and five negative items. Total scores range from 10 to 40, with higher scores representing lower self-esteem. The scale has demonstrated good psychometric properties including good test-retest reliability (e.g., Schmitt \& Allik, 2005) and high internal consistency (Cronbach's $\alpha=.81$ ) in a study that looked at the scale, Schmitt \& Allik, 2005).

Satisfaction with Life Scale (SWLS). The SWLS (Diener, Emmons, Larsen, \& Griffin, 1985) is a 5-item self-report measure of overall satisfaction with life, with higher scores indicting higher levels of satisfaction. Questions are global rather than specific in nature. The scores for this scale range from 5 to 35 , with a score of 20 representing a neutral point on the scale. Scores between 5 and 9 indicate the respondent is extremely dissatisfied with life; scores between 31 and 35 indicate the respondent is extremely satisfied. The scale has demonstrated high internal consistency (Cronbach's $\alpha=.92$; Glaesmer, Grande, Braehler, \& Roth, 2011), test-retest reliability ( $r=.82, \alpha=.87$; Diener et al., 1985), and convergent validity ( $r=.73$ between SWLS and a single-item measure of life satisfaction; Jovanovic, 2016).

Self-Compassion Scale-Short Form (SCS-SF). The SCS-SF (Raes, Pommier, Neff, \& Van Gucht, 2011) is a shortened (12-item), yet reliable and structurally equivalent version of the longer 36-item version of the scale (Neff, 2003; Raes et al., 2011). Items are rated using a Likerttype scale from 1 (almost never) to 5 (almost always), with questions designed to determine how respondents perceive their behaviours toward themselves during difficult times. The scale includes six facets (three components and their polar contrasts) of self-compassion: SelfKindness versus Self-Judgment; Common Humanity versus Isolation; and Mindfulness versus Over-Identification. The mean total scores for the SCS-SF range from 12-60, with a normative sample of undergraduate students averaging a mean of 36 (Raes et al., 2011). Psychometric properties of the SCS have been validated recently (Neff, 2016).

Five-Facet Mindfulness Questionnaire (FFMQ). The FFMQ (Baer, Smith, Hopkins, 
Krietemeyer, \& Toney, 2006) is a widely used 39-item inventory that assesses five facets of mindfulness including Observe, Describe, Act with Awareness, Nonjudge and Nonreact. Items are rated on a 5 -point scale $(1=$ never or very rarely true, to, $5=$ very often or always true $)$. Each facet's resulting score can range from 8 to 40, with the exception of Nonreact, which ranges from 7 to 35. The FFMQ has good psychometric properties including good internal consistencies for all facets (Cronbach's $\alpha$ of .67-.93 for the five subscales; Baer et al., 2006). As well, the FFMQ shows good discriminate validity, with meditators scoring higher on all facets, compared to nonmeditators (de Bruin, Topper, Muskens, Bogels, \& Kamphuis, 2012; Lilja et al., 2011). Testretest reliability for the FFMQ has not been assessed; however, the properties for a previous version of the measure (Kentucky Inventory of Mindfulness Skills; Baer, Smith, \& Allen, 2004) were 0.65 for the Observing scale and 0.83 for the Nonjudge scale.

\section{Data Analyses}

Data was first cleaned and checked for normality. Three variables (BDI-II, SAS-SR, and SWLS) were significantly skewed and $\log$ transformed to normalize. After log transformation, the SAS-SR and SWLS were adequately corrected for normality. The BDI-II was still significantly skewed at $-.70(\mathrm{SE}=.21)$. Following checks for normality, assumptions required for correlation analysis (absence of outliers, normality of variables, linearity, and homoscedasticity), and linear regression (normality of variables, linearity, homoscedascity, no multicollinearity) were all examined and deemed satisfactory. These assumptions also met requirements for mediation analysis.

Pearson's correlations were conducted to examine associations between levels of selfcompassion, mindfulness, and the clinical measures (LSAS, SPIN, BDI-II, SAS-SR, SWLQ, RSES). After correlational analyses, a series of multiple linear regressions were performed to determine the relative ability of self-compassion and facets of mindfulness to predict the clinical measures. The SCS-SF and facets of the FFMQ were force entered as predictors in the regression models, and each clinical measure was entered separately as dependent variables. Dominance analysis was then conducted using the RLM macro for SPSS (Darlington \& Hayes, 2017) to examine whether self-compassion or any of the five facets of mindfulness was the stronger predictor of each clinical measure.

To further examine the connections between mindfulness, self-compassion, and SAD, mediation analyses were conducted to examine if the five facets of mindfulness mediated the 
relationship between self-compassion and severity of social anxiety symptoms. A parallel multiple mediation with all five mediators (i.e., five facets of mindfulness) in the model was conducted on the LSAS and SPIN. Exploratory mediation analysis was also performed on each of the secondary clinical measures (BDI-II, RSES, SAS-SR, SWLS). A parallel multiple mediation approach estimates the indirect effects of several mediator variables in one mediation regression analysis, with the indirect effect equaling the amount by which the total effect of selfcompassion on a clinical measure drops when the mediators of mindfulness are included. The PROCESS for SPSS macro (Hayes, 2013) was used along with Preacher \& Hayes' (2008) bootstrapping method based on 1000 samples to obtain $95 \%$ bias-corrected confidence intervals. Due to our relatively small sample size and the number of mediators a series of mediation analyses with each mediator (i.e., each facet of mindfulness) separately were also examined to help ensure that Type II error did not occur in the multiple mediation analyses. The bootstrapping method is robust and results were consistent with the confirmatory single mediation analyses, so only parallel multiple mediation results are presented herein.

Results are based on 136 participants, except for the RSES and SWLS, which are based on 97 participants. Results were considered significant if $\mathrm{p} \leq .05$.

\section{Results}

\section{Participant characteristics}

Of the 136 participants included in this study, 50.7\% were female. Participants ranged in age from 18 to 71 years $(\mathrm{M}=40.54, \mathrm{SD}=14.14)$. The majority were Caucasian $(74.3 \%)$, married (56\%), and had at least postsecondary education (75\%). Table 1 shows the mean scores for the baseline clinical variables, the SCS-SF and the FFMQ facets. Examination of the clinical measures indicated that participants had marked to severe symptoms of SAD, mild depressive symptoms, and lower levels of self-esteem and social adjustment relative to normative samples (Sinclair et al., 2010; Weissman et al., 2001). Mean SWLS scores reflected the "neutral" range as outlined by previous researchers (Pavot \& Diener, 1993). Scores on the SCS-SF fell well below the mean found in a community sample $(M=78.33$; Raes et al., 2011), and the range of scores for the FFMQ (M range = 18.21-23.29) fell slightly below the range found in a sample of nonmeditators (M range =22.07-28.08; de Bruin et al., 2012).

\section{Correlation analyses}

Table 2 shows bivariate correlations between baseline clinical measures of SAD, self- 
compassion, and mindfulness. Analysis revealed that the SCS-SF and FFMQ facets were positively and significantly related, with correlation coefficients ranging from .22 (FFMQ Describe) to .56 (FFMQ Nonreact). Correlations between the SCS-SF and clinical measures of SAD indicated that higher levels of self-compassion were moderately associated with less severe social anxiety and depressive symptoms, and better social adjustment, satisfaction with life, and self-esteem. Analysis of the FFMQ revealed that with the exception of Observe, facets of mindfulness were significantly associated with less severe social anxiety and depressive symptoms and higher self-esteem. In addition, the FFMQ facets Act with Awareness and Describe were significantly associated with better social adjustment, while the FFMQ facet Act with Awareness was related to better satisfaction with life. 
Table 1 Descriptive statistics for study measures

\begin{tabular}{|c|c|c|}
\hline Measure & Mean & $\mathrm{SD}$ \\
\hline LSAS & 75.71 & 18.85 \\
\hline SPIN & 43.18 & 10.04 \\
\hline BDI-II & 12.81 & 9.81 \\
\hline SAS-SR & 2.17 & .42 \\
\hline SWLS & 18.10 & 7.56 \\
\hline RSES & 15.84 & 5.06 \\
\hline SCS-SF & 30.02 & 7.80 \\
\hline \multicolumn{3}{|l|}{ FFMQ } \\
\hline Awareness & 24.04 & 6.34 \\
\hline Describe & 22.85 & 6.64 \\
\hline Nonjudge & 23.29 & 5.73 \\
\hline Nonreact & 18.21 & 4.52 \\
\hline Observe & 23.54 & 5.79 \\
\hline
\end{tabular}

BDI-II = Beck Depression Inventory - II, FFMQ $=$ Five-Facet Mindfulness Questionnaire, LSAS = Liebowitz Social Anxiety Scale, RSES = Rosenberg Self-Esteem Scale, SAS-SR = Social Adjustment Scale - Self-Report, SCS-SF $=$ Self-Compassion Scale - Short Form, SD $=$ standard deviation, SPIN = Social Phobia Inventory, SWLS = Satisfaction With Life Scale. 
Table 2 Bivariate correlations between mindfulness, self-compassion, and clinical measures

\begin{tabular}{|c|c|c|c|c|c|c|c|}
\hline & LSAS & SPIN & BDI-II & SAS-SR & SWLS & RSES & SCS-SF \\
\hline \multicolumn{8}{|l|}{ FFMQ } \\
\hline Awareness & $-.23 *$ & $-.29 * *$ & $-.51 * *$ & $-.40 * *$ & $.25^{*}$ & $.49 * *$ & $.44 * *$ \\
\hline Describe & $-.31 * *$ & $-.25 *$ & $-.24 *$ & $-.20 *$ & .10 & $.23^{*}$ & $.22 *$ \\
\hline Nonjudge & $-.20 *$ & $-.23 *$ & $-.35^{* *}$ & -.16 & $.24 *$ & $.36^{* *}$ & $.41 * *$ \\
\hline Nonreact & $-.22 *$ & $-.25^{*}$ & $-.28 * *$ & -.23 & $.32 * *$ & $.37 * *$ & $.56^{* *}$ \\
\hline Observe & -.11 & -.09 & -.14 & -.18 & .28 & .06 & $.24 *$ \\
\hline SCS-SF & $-.42 * *$ & $-.43 * *$ & $-.47 * *$ & $-.41 * *$ & $.62 * *$ & $.65^{* *}$ & - \\
\hline
\end{tabular}

BDI-II = Beck Depression Inventory - II, FFMQ = Five-Facet Mindfulness Questionnaire, LSAS = Liebowitz Social Anxiety Scale, RSES = Rosenberg Self-Esteem Scale, SAS-SR = Social Adjustment Scale - Self-Report, SCS-SF = Self-Compassion Scale - Short Form, SPIN = Social Phobia Inventory, SWLS = Satisfaction With Life Scale.

Note: A low score on the SAS-SR indicates better social functioning.

$* p<.05$.

$* * p<.001$.

\section{Regression analysis}

Forced entry linear regression analyses were conducted to determine the relative importance of self-compassion and facets of mindfulness in predicting baseline clinical variables. Linear regressions for the social anxiety scales revealed that the full regression model was significant for the $\operatorname{LSAS}\left(F(6,129)=6.15, p<.001\right.$, with $\left.R^{2}=.24\right)$ and $\operatorname{SPIN}(F(6,129)=6.67, p$ $<.001$, with $R^{2}=22.2 \%$ ). Examination of the standardized beta coefficients (Table 3 ) revealed that the SCS-SF and FFMQ facet Describe were significant predictors in the model for both social anxiety measures. Analysis of the other baseline measures revealed that the full regression model was significant for the BDI-II $\left(F(6,122)=9.84, p<.01\right.$, with $\left.R^{2}=.33\right)$, $\operatorname{RSES}(\mathrm{F} F(6,90)=$ $14.84, p<.001$, with $\left.R^{2}=.50\right)$, SAS-SR $\left(F(6,129)=6.38, p<.001\right.$, with $\left.R^{2}=.23\right)$, and SWLS $\left(F(6,90)=9.77, p<.001\right.$, with $\left.R^{2}=.39\right)$. Inspection of the standardized beta-weights (Table 4) revealed that the SCS-SF and FFMQ facet Act with Awareness were significant predictors in the 
model for the BDI-II and SAS-SR; the SCF-SF and FFMQ facet Observe were significant predictors in the model for the SWLS; and the SCS-SF was the only significant predictor in the model for the RSES. For each clinical variable, subsequent dominance analysis revealed a value of 1.00 for self-compassion and 0.00 for each facet of mindfulness, indicating that selfcompassion dominated as a predictor of these baseline clinical measures over any of the facets of mindfulness.

Table 3 Prediction of social anxiety severity based on self-compassion and mindfulness

\begin{tabular}{lccccc}
\hline Measure & B & SE & $\beta$ & $t$ & $P$ \\
\hline LSAS & & & & & \\
SCS-SF & -.94 & .24 & -.39 & -3.87 & $.000^{* *}$ \\
FFMQ & & & & & \\
Awareness & .03 & .27 & .01 & .09 & .93 \\
Describe & -.77 & .25 & -.27 & -3.04 & $.003^{*}$ \\
Nonjudge & -.14 & .29 & -.04 & -.47 & .64 \\
Nonreact & .16 & .41 & .04 & .38 & .71 \\
Observe & .29 & .30 & .09 & .98 & .33
\end{tabular}

\section{SPIN}

SCS-SF

FFMQ

\begin{tabular}{|c|c|c|c|c|c|}
\hline Awareness & -.12 & .15 & -.07 & -.78 & .44 \\
\hline Describe & -.28 & .14 & -.19 & -2.09 & $.04 *$ \\
\hline Nonjudge & -.06 & .16 & -.04 & -.40 & .69 \\
\hline Nonreact & -.01 & .22 & -.003 & -.03 & .98 \\
\hline Observe & .17 & .16 & .10 & 1.06 & .29 \\
\hline
\end{tabular}


Table 4 Prediction of secondary clinical variables by self-compassion and mindfulness

\begin{tabular}{lllllll}
\hline Measure & B & SE & $\beta$ & $t$ & $P$ \\
\hline
\end{tabular}

\section{BDI-II}

SCS-SF

$-.02$

.005

$-.42$

$-4.35$

$.000 * *$

FFMQ

Awareness

$-.02$

.006

$-.27$

$-2.99$

$.003^{*}$

Describe

$-.01$

.005

$-.10$

$-1.14$

.26

Nonjudge

$-.004$

.006

$-.06$

$-.75$

.46

Nonreact

.02

.008

.19

1.93

.057

Observe

.000

.006

$-.002$

$-.02$

.98

\section{SAS-SR}

SCS-SF

$$
-.004
$$

.001

$-.33$

$-3.30$

$.001 * *$

FFMQ

Awareness

$-.004$

.001

$-.27$

$-2.96$

$.004 *$

Describe

$-.001$

.001

$-.06$

$-.67$

.51

Nonjudge

.001

.001

.08

.84

.40

Nonreact

.001

.002

.04

.38

.71

Observe

.001

.001

.04

.38

.70

\section{SWLS}

SCS-SF

$$
.02
$$

.003

.69

5.83

$.000^{* *}$

FFMQ

$\begin{array}{lccccc}\text { Awareness } & -.002 & .003 & -.05 & -.53 & .60 \\ \text { Describe } & .003 & .003 & .10 & 1.04 & .30 \\ \text { Nonjudge } & .000 & .003 & .01 & .06 & .95\end{array}$




$\begin{array}{llllll}\text { Nonreact } & -.002 & .005 & -.05 & -.45 & .66 \\ \text { Observe } & -.01 & .003 & -.20 & -2.13 & .04 *\end{array}$

\section{RSES}

SCS-SF

.40

.07

.61

5.67

$.000^{* *}$

FFMQ

Awareness

.14

.08

.17

1.83

.07

Describe

.11

.07

.13

1.59

.12

Nonjudge

.05

.08

.06

.68

.50

Nonreact

$-.09$

.12

$-.08$

$-.75$

.45

Observe

$-.12$

.08

$-.14$

$-1.60$

.11

BDI-II = Beck Depression Inventory - II, FFMQ = Five-Facet Mindfulness Questionnaire, RSES = Rosenberg Self-Esteem Scale, SAS-SR = Social Adjustment Scale - Self-Report, SCS-SF $=$ SelfCompassion Scale - Short Form, SWLS = Satisfaction With Life Scale.

Note: A low score on the SAS-SR indicates better social functioning

$* p<.05$.

$* * p<.001$.

Mediation analyses. The primary mediation of interest was whether the facets of mindfulness mediated the relationship between self-compassion and severity of social anxiety. Parallel mediation analysis indicated that self-compassion was indirectly related to the clinicianrated LSAS through its relationship with the FFMQ facet Describe (see Table 5 and Figure 1a and 1b). A 95\% bias-corrected confidence interval based on 1000 bootstrap samples (Preacher \& Hayes, 2008) indicated that the indirect effect through the Describe facet was entirely below zero $(b=-.15,95 \% \mathrm{CI}=-.34,-.02)$. All the other investigated indirect pathways had the value of 0 included in the $95 \%$ confidence interval and were not considered significant mediating pathways (see Table 5). Exploratory parallel mediation analysis of the relationship between selfcompassion and the BDI-II, RSES, SAS-SR, and SWLS did not reveal any significant mediating pathways. Based on these results, it appears that only the Describe facet, and not any of the other facets of mindfulness, partially mediates the relation between self-compassion and clinicianrated SAD symptom severity. 
Table 5 Self-compassion as predictor of SAD symptoms (LSAS and SPIN), mediated by facets of mindfulness

\begin{tabular}{|c|c|c|c|c|c|c|}
\hline \multirow[b]{2}{*}{ Variable } & \multicolumn{3}{|c|}{ LSAS } & \multicolumn{3}{|c|}{ SPIN } \\
\hline & $\begin{array}{c}\text { Point } \\
\text { estimate }\end{array}$ & $\begin{array}{l}\text { Boot } \\
\text { LLCI }\end{array}$ & $\begin{array}{l}\text { Boot } \\
\text { ULCI }\end{array}$ & $\begin{array}{c}\text { Point } \\
\text { estimate }\end{array}$ & $\begin{array}{l}\text { Boot } \\
\text { LLCI }\end{array}$ & $\begin{array}{l}\text { Boot } \\
\text { ULCI }\end{array}$ \\
\hline Total effect & $-1.02 * *$ & & & $-.55 * *$ & & \\
\hline Direct effect & $-.94 * *$ & & & $-.47 * *$ & & \\
\hline \multicolumn{7}{|c|}{ Indirect effects } \\
\hline Awareness & .01 & -.18 & .18 & -.04 & -.14 & .07 \\
\hline Describe & -.15 & -.34 & -.02 & -.05 & -.13 & .00 \\
\hline Nonjudge & -.04 & -.23 & .13 & -.02 & -.11 & .06 \\
\hline Nonreact & .05 & -.22 & .35 & .00 & -.16 & .17 \\
\hline Observe & .05 & -.04 & .19 & .03 & -.02 & .10 \\
\hline
\end{tabular}

Note: Indirect effects with confidence intervals that do not include zero are significant at the .05 level.

$* * p<.001$.

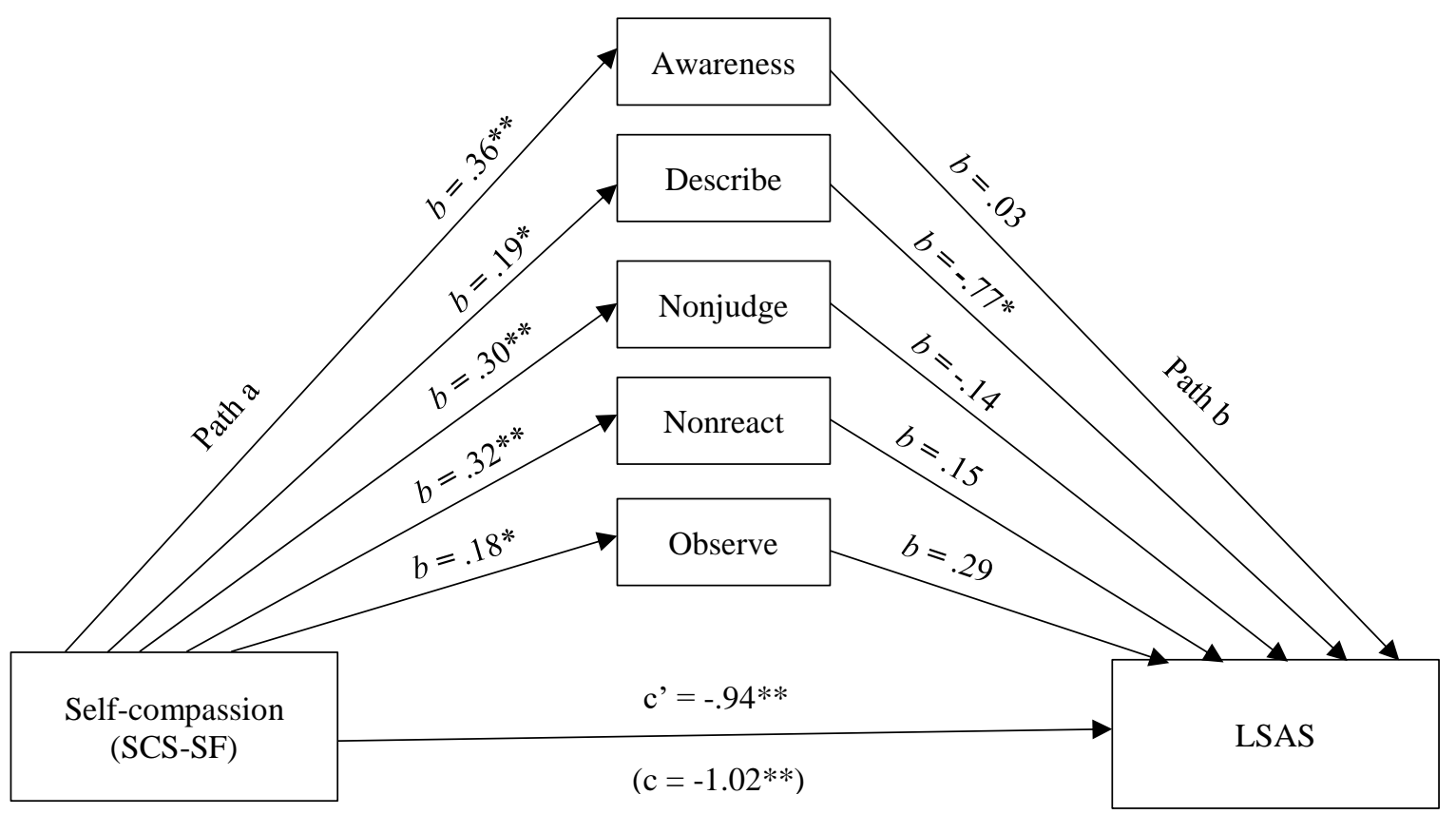

Fig. 1a Mediation between self-compassion and clinician-rated social anxiety severity (LSAS), with the five facets of mindfulness as partial parallel mediators.

$* p<.05, * * p<.001$. 


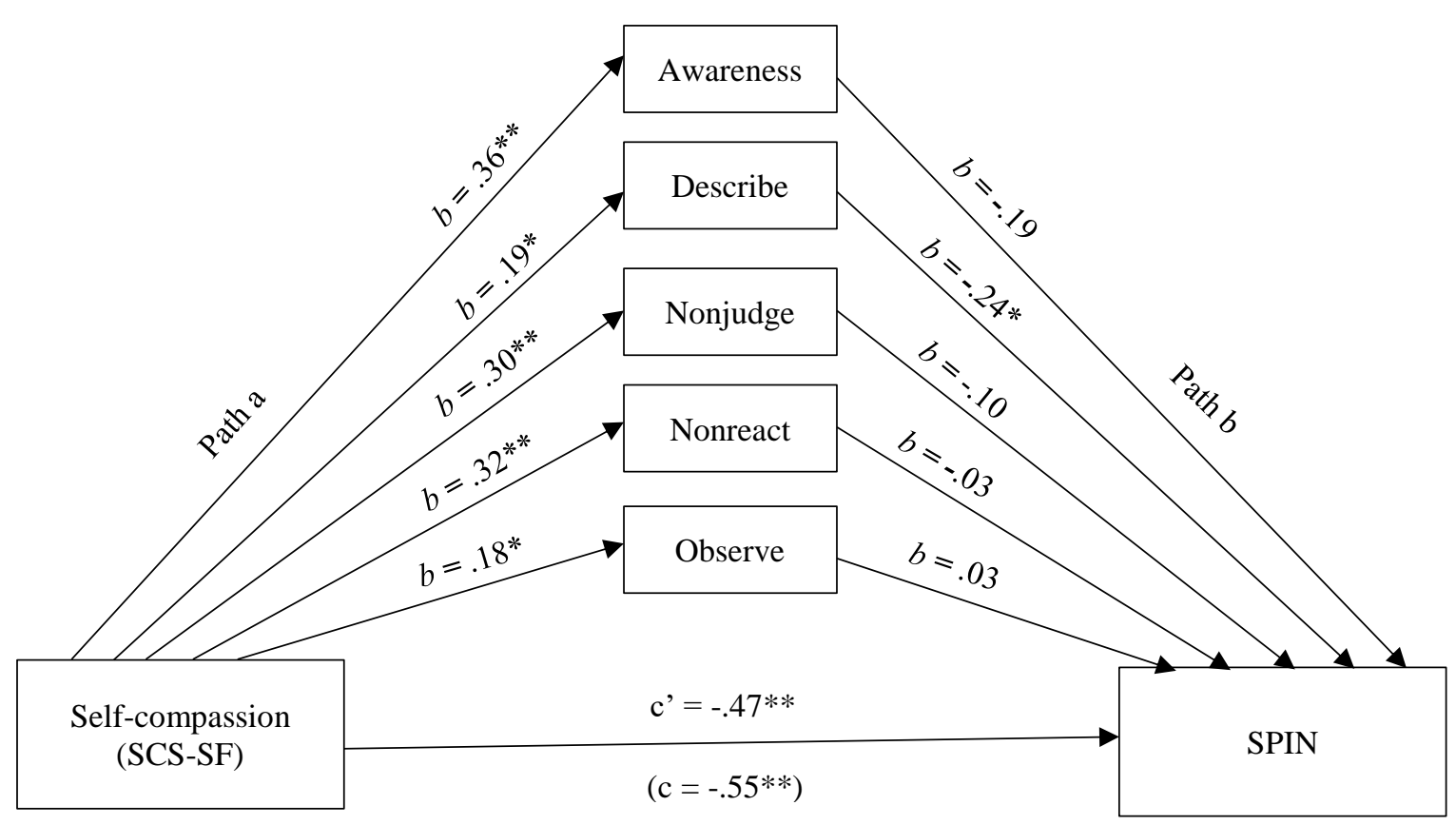

Fig. 1b Mediation between self-compassion and self-report social anxiety severity (SPIN), with the five facets of mindfulness as potential mediators.

$* p<.05, * * p<.001$.

\section{Discussion}

Self-compassion and mindfulness are related but distinct constructs that are thought to influence a variety of mental health outcomes. Few studies have evaluated these constructs concurrently in the context of mental health, and to our knowledge, no study has examined these constructs together in individuals with SAD. The present study examined the connections between mindfulness, self-compassion, and baseline clinical characteristics in SAD. As expected, participants in the current study had lower levels of self-compassion compared to a normative sample (Raes et al., 2011) and lower levels of mindfulness compared to a sample of non-meditators (de Bruin et al., 2012). Bivariate correlations revealed that self-compassion and the five facets of mindfulness measured by the FFMQ were significantly and positively correlated, with the strongest associations found for the facets Nonreact, Act with Awareness, and Nonjudge. Our findings concur with previous research in community samples that showed trait self-compassion and mindfulness are overlapping yet distinct constructs (Birnie et al., 2010; Hollis-Walker \& Colosimo, 2011; López et al., 2016), and provide unique data on the connection between these two constructs in individuals with SAD. 
Intercorrelations between self-compassion, mindfulness and baseline clinical variables indicate that self-compassion and facets of mindfulness influence variation in severity of SAD symptoms and other characteristics of the disorder. Moderate to strong associations were found between self-compassion and each of the clinical variables, with those higher in self-compassion reporting less severe symptoms of social anxiety and depression, less impairment, and higher self-esteem and satisfaction with life. Our findings are consistent with those of Blackie and Kocovski (2017) who reported a negative association between self-compassion and severity of fear of social interactions in participants seeking treatment for social anxiety and shyness. Although Werner et al. (2012) did not find an association between self-compassion and severity of SAD symptoms, they did find a negative association between self-compassion and fear of positive and negative evaluation. Other studies that examined clinical and non-clinical populations have found a similar association between self-compassion and psychological distress (MacBeth \& Gumbley, 2012; Van Dam et al., 2011).

Intercorrelations between the FFMQ facets and clinical variables were generally significant and small to moderate in size, depending on the mindfulness skill, and generally support findings that higher levels of trait mindfulness are linked with better psychological functioning (Baer et al., 2006; López et al., 2016). The strongest associations were between the FFMQ facet Act with Awareness, which denotes the ability to pay attention in the present moment and avoid automatic pilot, and depression severity ( $\mathrm{r}=-.51)$, self-esteem $(\mathrm{r}=.49)$ and social adjustment $(\mathrm{r}=-.40)$. The moderately strong negative association between Act with Awareness and depression is consistent with previous work that showed this specific mindfulness skill may be especially relevant for depression (Baer et al., 2006; Cash \& Whitingham, 2010; Curtiss \& Klemanski, 2014; López et al., 2016; Royuela-Colomer \& Calvete, 2016), possibly because it facilitates disengagement from dysphoric mood and cognitions and engagement in adaptive self-regulatory processes and behaviours. This mindfulness skill may also be relevant to self-esteem and social adjustment through similar mechanisms.

One finding of note is that the FFMQ facet Observe did not significantly correlate with any of the clinical variables, although it was a significant predictor of satisfaction with life in the linear regression model. A lack of association between this facet of mindfulness and psychological measures has been reported in some (Baer et al., 2008; Desrosiers, Klemanski, \& Nolen-Hoeksema, 2013; de Bruin et al., 2012) but not all studies (López et al., 2016). Baer et al 
(2006) suggested that the strength of the relationship between the facet Observe and psychological outcomes is likely influenced by meditation experience, with nonmeditating samples showing no significant association between this facet and psychological measures. As participants in the current sample were nonmeditators when they were screened for the clinical trials, this may explain the lack of significant correlations between this mindfulness skill and the clinical variables.

Results of the regression analyses showed that self-compassion was the more salient predictor of the clinical variables than was mindfulness and suggest that self-compassion may be more protective than mindfulness in socially anxious individuals and an important target for therapeutic interventions. Other researchers have also found that self-compassion is a better predictor of psychological health than mindfulness. For example, Van Dam et al. (2011) found that self-compassion was a more robust predictor of psychological health than mindfulness in a community sample of individuals seeking help for anxiety, and Woodruff et al. (2014) reported that self-compassion demonstrated a stronger ability than mindfulness to predict variance in psychological health in a non-clinical sample of undergraduate students. Similarly, Woods and Proeve (2014) found that self-compassion was a better predictor of shame-proneness than mindfulness. On the other hand, López et al. (2014) found that self-compassion and mindfulness predicted a similar amount of variance in measures of psychological distress in a large sample of community adults, and that mindfulness was a more robust predictor of positive affect than selfcompassion. Baer, Lykins and Peters (2012) found that the FFMQ facets and self-compassion predicted a similar amount of variance in wellbeing, however when total FFMQ scores were used in the mediation model, self-compassion emerged as a stronger predictor of wellbeing. Overall, these findings indicate that differences in methodology and measurement of mindfulness and self-compassion likely account for mixed findings and further research is needed to confirm if self-compassion and mindfulness differently influence psychological health in clinical and non-clinical samples.

Our finding that self-compassion may be a better predictor of the clinical features of SAD may be understood and explained by examining core features of the disorder along with aspects that define self-compassion. For those with SAD, the construct of self-compassion seems like a natural fit to predict symptoms that are often described as judgmental and self-critical. Gilbert and Miles (2000) posit that self-criticism triggers anxious responses, and that self-criticism can 
lead to shame if the individual believes that the critical beliefs are valid. Self-criticism is higher in individuals with SAD compared to those with other anxiety disorders and no psychiatric disorder (Cox et al., 2004) and predicts severity of social anxiety symptoms (Iancu et al., 2015). A study designed to improve symptoms of SAD found that changes in self-criticism were associated with positive outcomes; as self-criticism lessened, so too did symptoms of SAD (Cox et al., 2002). Part of Neff's (2003) conceptualization of self-compassion involves showing kindness toward oneself as opposed to responding with harsh self-criticism, especially in the face of failure. By addressing the self-critical aspect of SAD, self-compassion, then, seems to be protective over symptom severity. The potential protective effect of self-compassion in SAD is substantiated by a recent experimental study of post-event processing in socially anxious undergraduates who completed an impromptu speech (Blackie \& Kocovski, 2017). Participants who were assigned to a brief self-compassion exercise had lower levels of post-event processing the day after the speech and were more willing to engage in future social situations than those who were assigned to the rumination or control conditions.

Negative biases common in those with SAD may also be linked to why self-compassion is protective. Previous studies have shown that individuals with SAD report thinking that most people evaluate themselves and others with the same critical and negative lens (Alden, Bieling, \& Wallace, 1994). This biased social perception involves overattending to possible indicators of negative evaluation in others (Rapee \& Heimberg, 1997) and placing much importance on the external evaluation of others (Rodebaugh, Holaway, \& Heimberg, 2004). On the other hand, selfcompassion may shift these negative assumptions and associated social vigilance toward more self-kindness. Neff (2003) found that self-compassion was associated with being equally kind to oneself and to others, whereas lower self-compassion was associated with the tendency to be kinder to others than oneself. Said another way, self-compassion is associated with a lessened tendency to assume others are judging as harshly as one may judge themselves.

Self-compassion may also be protective for individuals with SAD due to its impact on fear responses to anxiety-provoking social stimuli. As noted earlier, Gilbert (2009) describes a three-part emotional regulation system, of which one part is a threat detection and protection system. This system prepares for threats, stimulating a fight-or-flight stress response. SAD involves an oversensitive threat detection system that scans for social threats (Bogels \& Mansell, 2004) and perceives threats or judgment from others (Clark \& Wells, 1995). States of negative 
self-evaluation, as often seen in those with SAD, have also been shown to be associated with stronger biological stress responses (Rohleder, Chen, Wolf, \& Miller, 2008). In addition to the threat detection and protection system, Gilbert's emotional regulation system theory highlights that another part of the regulation system, the soothing system, regulates and calms in the absence of threats. If the threat detection system becomes overactive and the soothing system underactive, further anxiety can result. Self-compassion, then, may benefit those with SAD by promoting this soothing system and rebalancing threat responses.

Gilbert and Irons (2005) proposed that self-compassion's encouragement of connection to others and self-kindness deactivates the threat system and associated autonomic arousal, and instead activates the soothing system and its oxytocin-opiate system. Other research indicates that higher levels of oxytocin are associated with feelings of interpersonal connectedness, safety, and trust (Feldman, Weller, Zagoory-Sharon \& Levine, 2007). Previous research has indicated that self-compassion is also linked to increased heart-rate variability, which in turn, is associated with a greater ability to self-soothe when stressed (Rockcliff et al., 2008). The benefit of selfcompassion for those with SAD may be partially found in its impact on the soothing system, rebalancing an overactive physiological threat response.

Mediation analyses revealed that with the exception of the facet Describe, none of the mindfulness facets mediated the relationship between self-compassion and severity of social anxiety. This is a surprising finding considering that mindfulness is closely connected to selfcompassion (Neff \& Dahm, 2014), self-compassion is a key process of change with mindfulnessbased interventions (Robins, Keng, Ekblad, \& Brantley, 2012; Evans, Wyka, Blaha \& Allen, 2018), and metacognitive skills operating during mindfulness may be essential to the development of self-compassion (Bergen-Cico \& Cheon, 2013). There are very few crosssectional studies that have explored mediational pathways between self-compassion, mindfulness and wellbeing and available data is based on non-clinical samples (Bluth \& Blanton, 2014) and therefore not generalizable to clinical populations. While the underlying mechanisms of selfcompassion and mindfulness in SAD requires more detailed investigation, the finding that the facet Describe was the only significant mediator in our model corroborates previous findings that this facet was the only significant predictor of behavioral avoidance, desired avoidance, and state anxiety responses to a social anxiety stressor (Parsons et al., 2017).

The mindfulness skill Describe involves labeling internal experiences with words. 
According to the disruption theory of language and emotion, labelling an emotional state or experience is thought to disrupt the intensity of that emotional state or experience (Lieberman, Inagaki, Tabibnia, \& Crockett, 2011) and Describing is theorized to be a detaching, emotion regulation strategy (Burklund, Creswell, Irwin, \& Lieberman, 2014). Previous research has revealed that affect labelling benefited participants with public speaking anxiety, with those who labelled their emotional state reporting less fear during a speech than those who did not (Niles, Craske, Lieberman, \& Hurr, 2015). Labeling emotions also promoted better progress with exposure therapy in participants with spider phobia, and interestingly, was more beneficial than other emotion regulation strategies like cognitive reappraisal and distraction. (Kircanski, Lieberman, \& Craske, 2012)

The facet of Describe may explain some of what connects self-compassion and severity of SAD symptom through its ability to tap into some of the major aspects self-compassion and SAD. Both self-compassion and Describe involve the capacity to distance oneself from maladaptive patterns of thinking. Research has shown that individuals with SAD believe that others will judge them with the same harsh criticism with which they judge themselves (Alden et al., 1994; Bogels \& Mansell, 2014) and selectively attend to visible social cues of judgment like frowning and signs of boredom to confirm these beliefs (Rapee \& Heimberg, 1997). Selfcompassion, on the other hand, involves seeing a common humanity between the self and others. Breines and colleagues (2015) studied dispositional self-compassion in response to immediate and repeated stressors and concluded that those who were more self-compassionate are less likely to assume a stressor is threatening. Labelling, too, helps foster a self-kind, non-threatening approach by not evaluating one's own thoughts or feelings, but rather describing and letting them pass.

Also, it may be that the ability to label one's experiences may help individuals with SAD to detach from problematic behavioural responses. Avoidance of social situations that trigger anxiety is a key component of SAD (Clark \& wells, 1995) and this avoidance reinforces feelings of anxiety and negative self-evaluation, helping to maintain the disorder. Past research has shown that labelling and describing are linked to a decrease in experiential avoidance for those with anxiety disorders (Curtiss \& Klemanski, 2014). In turn, decreases in experiential avoidance are associated with lower SAD symptomology (Kashdan et al., 2014).

Describing a thought or feeling with words may explain some of self-compassion's 
impact on SAD symptoms on a neural level. Labelling affect has been associated with a soothing of the amygdala, the part of the brain that detects threat and prepares for fight or flight (Torrisi, Lieberman, Bookheimer, \& Altshuler, 2013). An earlier study echoed these findings, suggesting that putting feelings into words encodes a negative emotional experience in a way that diminishes the emotional reactive response at the amygdala level through the right ventrolateral prefrontal cortex and medial prefrontal cortex (Lieberman, Eisenberger, Crockett, Tom, Pfeifer, $\&$ Way, 2007). The amygdala and associated threat systems are also deactivated and soothed when one practices a compassion-based meditation (Lutz, Brefczynski-Lewis, Johnstone, \& Davidson, 2008). In another study where participants were instructed to be either self-critical or self-reassuring in response to a given scenario, those who were self-assuring demonstrated increased activation of the ventrolateral prefrontal cortex (Longe, Maratos, Gilbert, Evans, Volker, Rockliff, \& Rippon, 2010).

It is notable that the same brain structures implicated in self-compassion and the facet Describe are also implicated in the pathophysiology of SAD. A neuroimaging study comparing emotional processing in patients with $\mathrm{SAD}$ and healthy controls found that the SAD group showed greater activation of fear-related limbic regions and the prefrontal cortex (Etkin \& Wager, 2007). A study of neural processes of negative self-beliefs in participants with SAD and healthy controls (Goldin, Manber-Ball, Werner, Heimberg, \& Gross, 2009) found that the SAD group had fewer soothing system brain areas activated during reappraisal of negative self-beliefs than the healthy control group. These finding supports the notion that SAD involves an imbalanced emotional regulation system, where the threat system is more active than the soothing system. Overall, neurobiological findings suggest that the same areas of the brain that are affected in socially anxious individuals are also activated in a soothing way when one is selfcompassionate and capable of labelling inner experiences.

Limitations of the study should be noted. Because the current study used cross-sectional data, causal claims regarding the relations between self-compassion, mindfulness, and clinical characteristics of SAD cannot be made. Future research may benefit from looking at how mindfulness and self-compassion mediate changes in SAD symptoms following treatment with mindfulness- and compassion-based interventions and exploring the temporal ordering of change using cross-lagged models. Another limitation of the study is the relatively small sample size, which may have reduced our ability to detect more significant findings. Finally, the data from 
this study were collected from volunteers who participated in randomized treatment trials and it is possible that results would differ if the sample comprised unselected individuals seeking standard care for SAD.

Despite these limitations, these preliminary findings contribute to knowledge of the interrelationship between trait mindfulness, self-compassion and SAD. Based on our findings, training in self-compassion and specific mindfulness skills may be particularly important to therapeutic interventions for the treatment of SAD. 


\section{Supplementary Mediation Analysis}

Further supplementary mediation analysis was conducted to evaluate a possible alternate direction of self-compassion as a mediator in the mindfulness-SAD symptom severity relationship. This analysis was not included in the research article owing to the exploratory nature of this analysis. A series of mediation analyses were conducted using Preacher \& Hayes' (2008) bootstrapping method based on 1000 samples to obtain bias-corrected confidence intervals. This method involved conducting a separate mediation analysis for each facet of mindfulness and each outcome variable (i.e., SAD clinical symptoms of LSAS and SPIN). Secondary clinical characteristics were also explored. The possibility of conducting path analysis using SPSS Amos was also considered; however, as both Amos and series of single mediations produced the same significant results, results are reported as a series of single mediations. Based on Preacher \& Hayes (2008) bootstrapping method, indirect effects were considered significant if the $95 \%$ CI did not include zero.

\section{Results}

For the mindfulness-SAD symptom severity relationship, results showed significant indirect effect of self-compassion for each facet of mindfulness (see Table 6 and Figure 2 and 3). The $95 \%$ bias-corrected confidence intervals based on 1000 bootstrap samples (Preacher \& Hayes, 2008) indicated that the indirect effect through self-compassion was entirely below zero for each facet of mindfulness on the LSAS (Awareness: $b=-.52,95 \% \mathrm{CI}=-.79,-.27$; Describe: $b=-.23,95 \% \mathrm{CI}=-.47,-.04$; Nonjudge: $b=-.55,95 \% \mathrm{CI}=-.88,-.29$; Nonreact: $b=-.1 .00$, $95 \% \mathrm{CI}=-.1 .47,-.57$; Observe: $b=-.33,95 \% \mathrm{CI}=-.66,-.08)$ and the SPIN (Awareness: $b=-$ $.26,95 \% \mathrm{CI}=-.47,-.11$; Describe: $b=-.13,95 \% \mathrm{CI}=-.29,-.02$; Nonjudge: $b=-.29,95 \% \mathrm{CI}=-$ $.48,-.14$; Nonreact: $b=-.52,95 \% \mathrm{CI}=-.84,-.25$; Observe: $b=-.18,95 \% \mathrm{CI}=-.25,-.04)$. Important to note are the non-significant direct effects of mindfulness on the LSAS and SPIN, with the exception of the facet of Describe $(b=-.64$ and $b=-.24$ for the LSAS and SPIN respectively, both $p<.05$ ). This means that Awareness, Nonjudge, Nonreact, and Observe do not significantly predict LSAS or SPIN values. As well, the total effect (i.e., the sum of the direct and indirect effects) for the Observe-SAD symptom severity relation is still non-significant ( $b=$ .35 and $b=-.15$ for the LSAS and SPIN respectively), though this relation does not require significance in order for the indirect effect to be significant. All other total effects are significant, meaning that adding the mediator of self-compassion to the relation takes it from non-significant 
to significant. Exploratory mediation analysis of the relationship between self-compassion and the BDI-II, RSES, SAS-SR, and SWLS revealed similar significant mediating pathways, with self-compassion mediating the relationship between facets of mindfulness and these secondary outcomes. Exceptions included analysis involving the facet of Describe and SWLS, and Describe and RSES; for these, the direct effect of Describe on the outcome variable $(b=.004, p=.89$ for SWLS and $b=.09, p=.17$ for RSES) was non-significant and the indirect effect of selfcompassion was still non-significant as both $95 \%$ confidence intervals included zero $(95 \% \mathrm{CI}=$ $-.0006, .01$ for SWLS; $95 \%$ CI = -.02, .23 for RSES).

Table 6 Facets of mindfulness as predictors of SAD symptoms (LSAS and SPIN), as mediated by self-compassion

\begin{tabular}{cccccccc}
\hline & \multicolumn{3}{c}{ LSAS } & \multicolumn{3}{c}{ SPIN } \\
\cline { 2 - 7 } Variable & Point & Boot & Boot & Point & Boot & Boot \\
& estimate & LLCI & ULCI & estimate & LLCI & ULCI
\end{tabular}

\section{Awareness}

Total effect $-.69 *$

$-.45 * *$

Direct

$-.18$

$-.19$

Indirect

$-.52$

$-.79$

$-.27$

$-.26$

$-.47 \quad-.11$

\section{Describe}

Total effect

Direct

Indirect

\section{Nonjudge}

Total effect

Direct

Indirect

\section{Tota}

$-.87 * *$

$-.64^{*}$

$-.23$

$-.47$

$-.04$

$-.13$

$-.38 *$

$-.24 *$

$\begin{array}{ll}-.29 & -.02\end{array}$

\section{Nonreact}




$\begin{array}{ccccccc}\text { Total effect } & -.93^{*} & & -.55^{*} & \\ \text { Direct } & .07 & & & -.03 & & \\ \text { Indirect } & -1.00 & -1.47 & -.57 & -.52 & -.84 & -.25\end{array}$

\section{Observe}

Total effect

Direct

Indirect

Note: Indirect effects with confidence intervals that do not include zero are significant at the .05 level. $* p<.05$.

a)

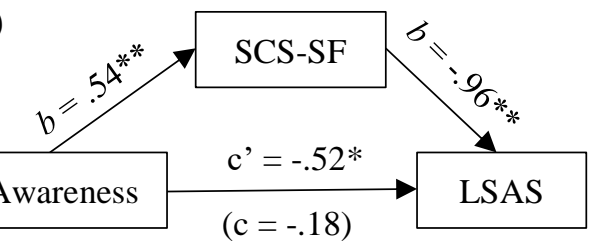

c)

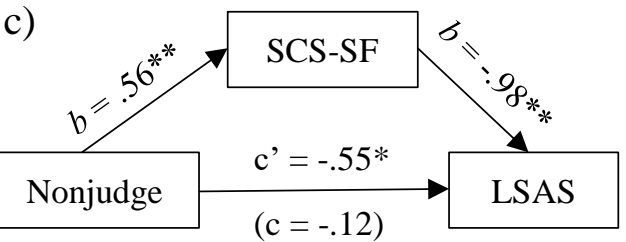

b)

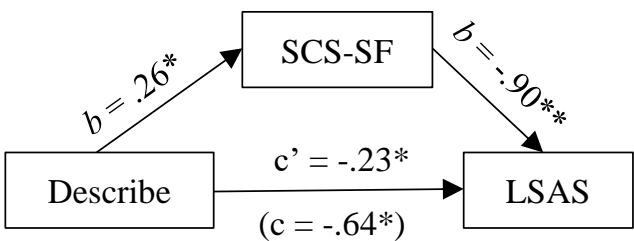

d)

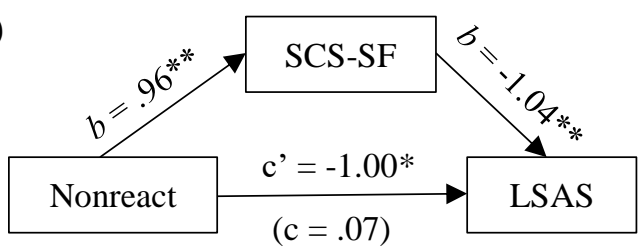

e)

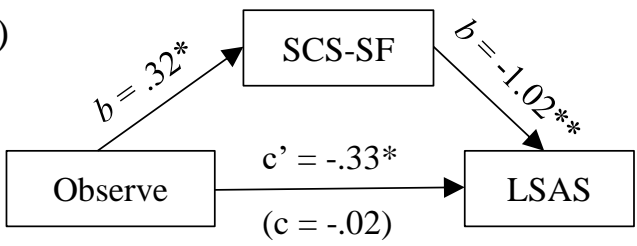

Fig 2 (a-e) Mediation analyses between each facet of mindfulness and clinicianreport social anxiety severity (LSAS), with self-compassion as potential mediator. $* p<.05, * * p<.001$. 
a)

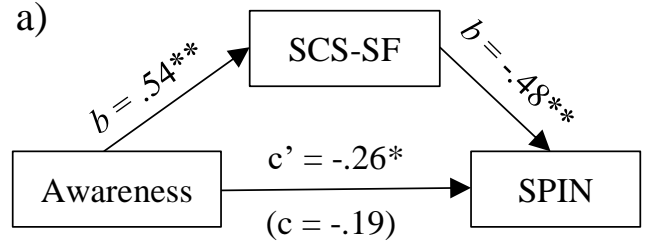

c)

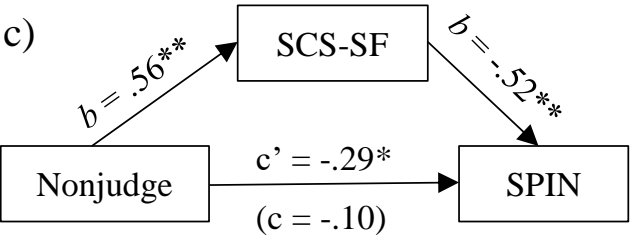

b)

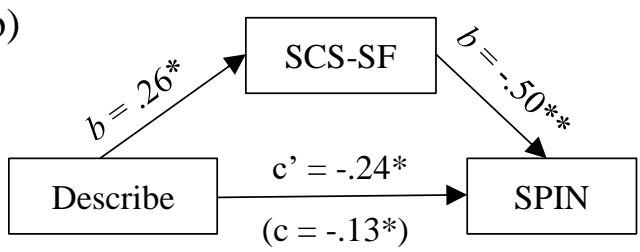

d)

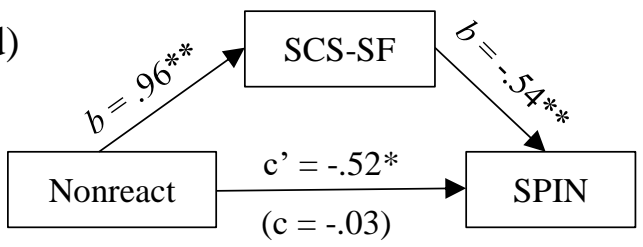

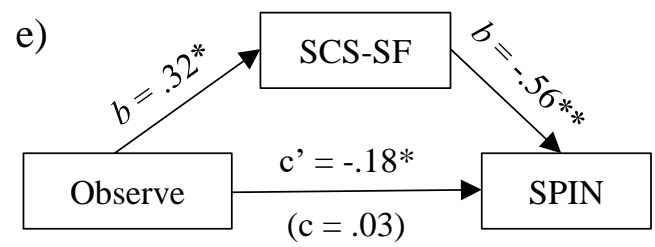

Fig 3 (a-e) Mediation analyses between each facet of mindfulness and self-report social anxiety severity (SPIN), with self-compassion as potential mediator. $* p<.05, * * p<.001$.

\section{Discussion}

Results of the additional exploratory analysis showed that self-compassion was an important mechanism in the relation between facets of mindfulness and severity of SAD symptoms. Highlighting this importance are the connections between the facets of Act with Awareness, Nonjudge and Nonreact and the LSAS and SPIN; these relations are only significant once self-compassion is included as an indirect effect. These results add to our understanding of the relationship between self-compassion, mindfulness, and social anxiety, again highlighting the role that self-compassion plays for individuals with social anxiety. As found in the analysis of self-compassion as a predictor and facets of mindfulness as mediators, self-compassion is particular important in relationships with social anxiety and related clinical characteristics. The results of the exploratory alternate direction of mediation also mirror the relative importance of self-compassion found in the mindfulness-as-mediator analysis and the regression analyses conducted. Other researchers have considered self-compassion a macro concept (Bergen-Cico and Cheon, 2016) and the current study's findings in both directions of mediation echo this conclusion. 


\section{General Discussion}

The aim of this thesis was to examine the connections between self-compassion, mindfulness, and clinical characteristics associated with SAD. In the first research question, the associations between the main concepts and the ability of self-compassion and facets of mindfulness to predict clinical characteristics was examined. In the next two research questions, the potential mechanisms underlying the connections between variables was considered by conducting mediation analyses in both directions (i.e., facets of mindfulness as potential mediators, and self-compassion as a potential mediator).

Results from the first research question showed consistent associations between selfcompassion and all clinical characteristic variables. For mindfulness, most of the facets showed associations with clinical characteristics, with the exception of Observe. These results are supported by past research that looked at associations between self-compassion and SAD (e.g., Werner et al., 2012) and associations between mindfulness and social anxiety symptoms (e.g., Parsons et al., 2017). Results of regression and dominance analyses suggested that selfcompassion was a better predictor of all clinical characteristics. Studies that have conducted similar analyses have also yielded similar results. For example, Woodruff et al. (2014) reported that self-compassion demonstrated a stronger ability than mindfulness to predict variance in psychological health in a non-clinical sample of undergraduate students, and Woods and Proeve (2014) found that self-compassion was a better predictor of shame-proneness than mindfulness.

In considering the second and third research questions, mediation analysis yielded results that further indicated the protective value of self-compassion for individuals with SAD. As a predictor, with facets of mindfulness as potential mediators, only Describe emerged as a significant partial mediator in the self-compassion-clinician-rated SAD symptom severity relationship. In the alternate direction, self-compassion was a significant mediator for every facet of mindfulness, acting as an important mechanism in the relationship between each facet of mindfulness and both clinician- and self-report SAD symptom severity. This is a surprising finding considering that mindfulness is closely connected to self-compassion (Neff \& Dahm, 2014), self-compassion is a key process of change with mindfulness-based interventions (Robins, Keng, Ekblad, \& Brantley, 2012; Evans, Wyka, Blaha \& Allen, 2018), and metacognitive skills operating during mindfulness may be essential to the development of self-compassion (BergenCico \& Cheon, 2013). Conducting the exploratory alternate direction of mediation was important 
in confirming the results of the initial mediation analysis and the ordering results, and highlighting the findings of self-compassion as more explanative in its relation to SAD and related clinical characteristics.

Results of the additional exploratory analysis indicated that the relation between facets of mindfulness and severity of SAD symptoms was mediated by self-compassion. The connection between the facets of Act with Awareness, Nonjudge and Nonreact and the LSAS and SPIN are only significant once self-compassion is included as an indirect effect. The direct effect of each of these facets on severity of SAD symptoms were non-significant. When the mediator of selfcompassion was added, the total effect was significant for each. Self-compassion was a significant mediator for the connections between the facets of Observe and Describe and symptom severity, though in unique ways.

The overarching importance of self-compassion is highlighted once again by the results of the exploratory supplementary analysis. Self-compassion explains much of the association between mindfulness and SAD symptom severity, for the most part taking the facet-SAD clinical symptom relation from non-significant to significant (e.g., Act with Awareness-SPIN association goes from a non-significant direct effect to a significant total effect with selfcompassion in the model). For three of the five facets of mindfulness (with Observe being the exception, discussed below), their effect on SAD clinical symptoms is only significant when self-compassion is included in the model. The facet of Describe showed a significant direct effect on both the LSAS and SPIN. With self-compassion in the model as a mediator, the total effect for both outcome variables were also significant. So, self-compassion is a partial mediator of the Describe-SAD clinical symptom association. Describe is more predictive of SAD clinical symptoms than the other facets, and self-compassion explains some of this predictive ability. These results indicating the benefits of the Describe facet are in line with previous research into the positive outcomes associated with labelling one's inner experiences (Niles, Craske, Lieberman, \& Hurr, 2015; Kircanski, Lieberman, \& Craske, 2012). As discussed above in the research article, both self-compassion and Describe involve the capacity to distance oneself from maladaptive patterns of thinking that are common in individuals with SAD (e.g., Alden et al., 1994; Bogels \& Mansell, 2014; Rapee \& Heimberg, 1997).

In contrast to the findings for the facet of Describe, the facet of Observe showed nonsignificant direct effects and total effects. So, while the indirect effect of self-compassion did 
partially mediate the relation between Observe and each measure of SAD symptom severity, it did not mediate enough to make the total effect significant. These findings are in line with our study's correlational results for the facet of Observe; this facet was the only one without any significant correlation to any of the clinical characteristic variables. The facet may align itself some of the common symptoms of SAD like self-focused attention, rumination, and selfcriticism (Bogels \& Mansell, 2004; Baer et al., 2006, 2008). Indeed, past research by Baer and colleagues (2008) found that the facet of Observe did not correlate significantly in the expected direction with psychological symptoms and psychological well-being in a sample of nonmeditators. de Bruin and associates (2012) found no correlation between the facet Observe and measures of worry and rumination, although they did find a positive relationship between Observe and thought suppression. The authors theorized that for some, the more one attends to internal and external sensations and emotions, the more self-critical they may be about these sensations and emotions. This explanation is especially relevant for the current study's population, as self-criticism and ruminative, negative self-talk are common in those with SAD (Cox, Walker, Enns, \& Karpinski, 2002; Vassilopoulos, 2008). So, for those who are not active meditators, as in our current sample, the trait of taking notice of each thought or feeling may encourage over-attention to negative cues.

Overall results of this study indicate the protective power of self-compassion for individuals with SAD. Self-compassion shows robust, consistent associations and ability to predict SAD symptoms: in general, the lower the baseline self-compassion, the higher the clinical symptoms of SAD. Further, self-compassion seems to be more protective than mindfulness for individuals with SAD, and self-compassion explains much of the effect between facets of mindfulness and clinical characteristics of SAD. Further, only the mindfulness facet of Describe acts as a mechanism in the relationship between self-compassion and clinician-rated SAD symptom severity. Supplementary mediation analyses also suggested that Describe has a unique role, as it was the only facet of mindfulness that had a significant direct effect (i.e., predictive relationship with SAD symptoms prior to including self-compassion into the model). While preliminary research has shown that targeting mindfulness in SAD treatment is effective (e.g., Koszycki et al., 2016), it may be that certain facets of mindfulness like the ability to describe and label passing thoughts may be especially related to self-compassion and its positive impact on those with SAD. 


\section{Implications}

Results of this research suggest that training in self-compassion and specific mindfulness skills may be particularly important to therapeutic interventions for the treatment of SAD. The facet of Describe in particular may represent an intersecting area of interest with selfcompassion: labelling an inner experience and allowing it to pass may be particularly protective for individuals with social anxiety. These findings add to an area of research that has yet to be fully explored, contributing insight into mindfulness and self-compassion and SAD. In the future, research may benefit from continuing to measure both mindfulness and self-compassion in SAD-based studies; presently, most research has measured one or the other, but not both. As well, intervention-based, longitudinal study designs may help determine the value of focusing on self-compassion and labelling in the treatment of SAD.

\section{Conclusion}

The results of the current study are consistent with existing evidence that indicates the benefits of self-compassion and mindfulness for psychological health. The results of the current study also suggest that self-compassion may be particularly protective over SAD symptom severity and related clinical characteristics. As the first study to consider self-compassion and mindfulness in individuals with $\mathrm{SAD}$, the results may spur future research to consider how treatment plans for SAD can better target self-compassion and the mindfulness skill of Describe. The current study's results are encouraging in that they consistently highlight the connection between self-compassion and SAD symptom severity, even at baseline for those who have yet to be treated.

Up to present, very few research studies have considered both self-compassion and mindfulness as comparative concepts in psychological disorders. The current study's findings of differing ability to predict symptoms indicate that future research may benefit from considering both measures, and, for SAD in particular, self-compassion, in their analyses. Other research may also benefit from using temporal ordering to explore causal pathways. 


\section{References}

Alden, L. E., Bieling, P. J., \& Wallace, S. T. (1994). Perfectionism in an interpersonal context: a self-regulation analysis of dysphoria and social anxiety. Cognitive Therapy and Research, 18, 297-316.

Allen, N. B., Chambers, R., Knight, W., \& Melbourne Academic Mindfulness Interest Group. (2006). Mindfulness-based psychotherapies: A review of conceptual foundations, empirical evidence and practical considerations. Australian \& New Zealand Journal of Psychiatry, 40, 285-94.

American Psychiatric Association (2013). Diagnostic and statistical manual of mental disorders (5th ed.). Arlington, VA: American Psychiatric Publishing.

Anicha, C. L., Ode, S., Moeller, S. K., \& Robinson, M. D. (2012). Toward a cognitive view of trait mindfulness: Distinct cognitive skills predict its observing and nonreactivity facets. Journal of Personality, 80, 255-285.

Baer, R. A. (2011). Measuring mindfulness. Contemporary Buddhism: Interdisciplinary Journal, 12, 241-261.

Baer, R. A., Smith, G. T., \& Allen, K. B. (2004). Assessment of mindfulness by self-report: the Kentucky inventory of mindfulness skills. Assessment, 11, 191-206.

Baer, R. A., Smith, G. T., Hopkins, J., Krietemeyer, J., \& Toney, L. (2006). Using self-report assessment methods to explore facets of mindfulness. Assessment, 13, 27-45.

Baer, R., Smith, G., Lykins, E., Button, D., Krietemeyer, J., Sauer, S.,... \& Williams, J. M. G. (2008). Construct validity of the Five Facet Mindfulness Questionnaire in meditating and nonmeditating samples. Assessment, 15, 329-342. doi: 10.1177/1073191107313003

Baron, R. M., \& Kenny, D. A. (1986). The moderator-mediator variable distinction in social psychological research: Conceptual, strategic and statistical considerations. Journal of Personality and Social Psychology, 51, 1173-1182.

Barnard, L. K., \& Curry, J. F. (2011). Self-compassion: Conceptualizations, correlates, \& interventions. Review of General Psychology, 15, 289-303.

Beck, A. T., \& Emery, G. (1985). Anxiety disorders and phobias: A cognitive perspective. New York: Basic Books.

Beck, A. T., Steer, R. A., \& Brown, G. K. (1996). Manual for the Beck Depression Inventory-II. San Antonio, TX: Psychological Corporation. 
Bergen-Cico, D. \& Cheon, S. (2013). The mediating effects of mindfulness and self-compassion on trait anxiety. Mindfulness, 5, 505-519. DOI: 10.1007/s12671-013-0205-y

Birnie, K., Speca, M., \& Carlson, L. E. (2010). Exploring self-compassion and empathy in the context of Mindfulness-based Stress Reduction (MBSR). Stress and Health, 26, 359-371.

Bishop, S. R., Lau, M., Shapiro, S., Carlson, L., Anderson, N. D., Carmody, J.,... \& Devins, G. (2004). Mindfulness: A proposed operational definition. Clinical Psychology: Science and Practice, 11, 191-206. doi: 10.1093/clipsy.bph077

Blackie, R. A., \& Kocovski, N. L. (2017). Examining the relationships among self-compassion, social anxiety, and post-event processing. Psychological Reports, Jan 1:33294117740138.

Bluth, K., \& Blanton, P. (2014). Mindfulness and self-compassion: Exploring pathways to adolescent emotional well-being. Journal of Child and Family Studies, 23, 1298-1309.

Bogels, S. M. \& Mansell, W. (2004). Attention processes in the maintenance and treatment of social phobia: Hypervigilance, avoidance and self-focused attention. Clinical Psychology Review, 24, 827-856.

Bohlmeijer, E., Prenger, R., Taal, E., \& Cuijpers, P. (2010). The effects of mindfulness-based stress reduction therapy on mental health of adults with a chronic medical disease: A meta-analysis. Journal of Psychosomatic Research, 68, 539-544. doi: 10.1016 /j.jpsychores.2009.10.005

Breines, J. G., \& Chen, S. (2012). Self-compassion increases self-improvement motivation. Personality and Social Psychology Bulletin, 38, 1133-1143. doi: 10.1177 /0146167212445599

Brown, K. W.\& Ryan, R. M. (2003). The benefits of being present: Mindfulness and its role in psychological well-being. Journal of Personality and Social Psychology, 84, 822-848.

Bullis, J. R., Boe, H. J., Asnaani, A., \& Hofmann, S. G. (2014). The benefits of being mindful: Trait mindfulness predicts less stress reactivity to suppression. Journal of Behavior Therapy and Experimental Psychiatry, 45, 57-66. doi: 10.1016/j.jbtep.2013.07.006

Burklund, L. J., Creswell, J. D., Irwin, M. R., \& Lieberman, M. D. (2014). The common and 
distinct neural bases of affect labeling and reappraisal in healthy adults. Frontiers in Psychology, 5.

Chiesa, A., \& Serretti, A. (2011). Mindfulness based cognitive therapy for psychiatric disorders: A systematic review and meta-analysis. Psychiatry Research, 187, 441-453.

Chavira, D.A., Stein, M.B., \& Malcarne, V.L. (2002). Scrutinizing the relationship between shyness and social phobia. Journal of Anxiety Disorders, 16, 585-598.

Clark, D.M., \& Wells, A. (1995). A cognitive model of social phobia. In R. G. Heimberg, M. R. Liebowitz, D. A. Hope, \& F. R. Schneier (Eds.), Social phobia: Diagnosis, assessment, and treatment (pp. 69-93). New York: Guilford Press.

Connor, K. M., Davidson, J. R., Churchill, L. E., Sherwood, A., Foa, E., \& Weisler, R. H. (2000). Psychometric properties of the Social Phobia Inventory (SPIN): New self-rating scale. British Journal of Psychiatry, 176, 379-386.

Cox, B. J., Fleet, C., \& Stein, M. B. (2004). Self-criticism and social phobia in the US national comorbidity survey. Journal of Affective Disorders, 82, 227-234. doi: 10.1016/j.jad.2003.12.012

Cox, B. J., Walker, J. R., Enns, M. W., \& Karpinski, D. C. (2002). Self-criticism in generalized social phobia and response to cognitive-behavioral treatment. Behavior Therapy, 33, 479491. doi: 10.1016/S0005-7894(02)80012-0

Darlington, R. B., \& Hayes, A. F. (2017). Regression analysis and linear models: Concepts, application, and implementation. New York: The Guilford Press.

de Bruin, E., Topper, M., Muskens, J.G., Bogels, S.M., \& Kamphuis, J.H. (2012). Psychometric properties of the Five Facets Mindfulness Questionnaire (FFMQ) in a meditating and a non-meditating sample. Assessment,19(2), 187-197.

Desrosiers, A., Klemanski, D. H., \& Holen-Hoeksema, S. (2013). Mapping mindfulness onto dimensions of anxiety and depression. Behavior Therapy, 44, 373-384.

Diener, E., Emmons, R. A., Larsen, R. J., \& Griffin, S. (1985). The Satisfaction With Life Scale. Journal of Personality Assessment, 49, 71-75.

Dozois, D. J. A., Dobson, K. S., \& Ahnberg, J. L. (1998). A psychometricevaluation of the Beck Depression Inventory-II. Psychological Assessment, 10, 83-89. 
Edwards, D., Yarvis, R., Mueller, D., Zingale, H., \& Wagman, W. (1978). Test-taking and the stability of adjustment scales: Can we Assess patient deterioration? Evaluation Review, 2, 275-291.

Etkin, A., \& Wager, T. D. (2007). Functional neuroimaging of anxiety: A meta-analysis of emotional processing in PTSD, social anxiety disorder, and specific phobia. American Journal of Psychiatry, 164, 1476-1488.

Evans, S., Wyka, K., Blaha, K. T., \& Allen, E. S. (2018). Self-compassion mediates improvement in well-being in a mindfulness-based stress reduction program in a community-based sample. Mindfulness. Advance online publication. doi: 10.1007 /s12671-017-0872-1

Feldman, R., Weller, A., Zagoory-Sharon, O., \& Levine, A. (2007). Evidence for a neuroendocrinological foundation of human affiliation plasma oxytocin levels across pregnancy and the postpartum period predict mother-infant bonding. Psychological Science, 18, 965-970.

First, M. B., Spitzer, R. L, Gibbon, M., \& Williams, J. B.W. (2002). Structured Clinical Interview for DSM-IV Axis I Disorders, Research Version. (SCID-I) New York: Biometrics Research, New York State Psychiatric Institute.

Fresco, D.M., Coles, M.E., Heimberg, R.G., Liebowitz, M.R., Hami, S., Stein, M.B., \& Goetz, D. (2001). The Liebowitz Social Anxiety Scale: A comparison of the psychometric properties of self-report and clinician-administered formats. Psychological Medicine, 31, 1025-1035.

Gilbert, P. (2009). Introducing compassion-focused therapy. Advances in Psychiatric Treatment, 15, 199-208. doi: 10.1192/apt.bp.107.005264

Gilbert, P., \& Irons, C. (2005). Focused therapies and compassionate mind training for shame and self-attacking. Compassion: Conceptualisations, research and use in psychotherapy, 263-325. 
Gilbert, P., \& Miles, J. N. (2000). Sensitivity to social put-down: Its relationship to perceptions of social rank, shame, social anxiety, depression, anger and self-other blame. Personality and Individual Differences, 29, 757-774.

Glaesmer, H., Grande, G., Braehler, E., \& Roth, M. (2011). The German version of the satisfaction with lifescale (SWLS). European Journal of Psychological Assessment, 27, 127-132.

Goldin, P. R., \& Gross, J. J. (2010). Effects of mindfulness-based stress reduction (MBSR) on emotion regulation in social anxiety disorder. Emotion, 10, 83-91.

Goldin, P. R., Morrison, A., Jazaieri, H., Brozovich, F., Heimberg, R., \& Gross, J. J. (2016). Group CBT versus MBSR for social anxiety disorder: A randomized controlled trial. Journal of Consulting and Clinical Psychology, 84, 427-437. doi: 10.1037/ccp0000092

Goyal, M., Singh, S., Sibinga, E. M. S., Gould, N. F., Rowland-Seymour, A., Sharma, R., Berger, Z., Sleicher, D., Maron, D. D., Shihab, H. M., Ranasinghe, P. D., Linn, S., Saha, S., Bass, E. B. \& Haythornthwaite, J. A. (2014). Meditation programs for psychological stress and well-being a systematic review and meta-analysis. Journal of the American Medical Association, 174, 357-368.

Grossman, P., Neimann, L., Schmidt, S., \& Walach, H. (2004). Mindfulness-based stress reduction and health benefits: A meta-analysis. Journal of Psychosomatic Research, 57, 35-43. doi: 10.1016/S0022-3999(03)00573-7

Guy, W. (Ed.). (1976). Clinical Global Impression (CGI). In ECDEU Assessment Manual for Psychopharmacology. Rockville, MD: U.S. Department of Health, Education, and Welfare

Hayes, A. F. (2013). Introduction to mediation, moderation, and conditional process analysis a regression-based approach. New York, NY: Guilford Press.

Heimberg, R.G., Horner, J.K., Juster, H.R., Safren, S.A., Brown, E.J., Schneier, R.F., \& Liebowitz, M.R. (1999). Psychometric properties of the Liebowitz Social Anxiety Scale. Psychological Medicine, 29, 199-212.

Hjeltnes, A., Molde, H., Schanche, E., Vollestad, J., Svendsen, J. L., Moltu, C., \& Binder, P.-E. (2017). An open trial of mindfulness-based stress reduction for young adults with social 
anxiety disorder. Scandinavian Journal of Psychology, 58, 80-90. doi:

10.1111/sjop. 12342

Hofmann, S. G., Sawyer, A. T., Witt, A. A., \& Oh, D. (2010). The effect of mindfulness-based therapy on anxiety and depression: A meta-analytic review. Journal of Consulting and Clinical Psychology, 78, 169-83.

Hollis-Walker, L., \& Colosimo, K. (2011). Mindfulness, self-compassion, and happiness in nonmeditators: A theoretical and empirical examination. Personality and Individual Differences, 50, 222-227. doi: 10.1016/j.paid.2010.09.033

Holt, C.S., Heimberg, R.G., Hope, D.A., \& Liebowitz, M.R., (1992). Situational demands of social phobia. Journal of Anxiety Disorders, 6, 63-77.

Hope, D. A., Rapee, R. M., Heimberg, R. G., \& Dombeck, M. J. (1990). Representations of the self in social phobia: Vulnerability to social threat. Cognitive Therapy and Research, 14, 177-189. doi: 10.1007/BF01176208

Iancu, I., Bodner, E., \& Ben-Zion, I. Z. (2015). Self-esteem, dependency, self-efficacy and selfcriticism in social anxiety disorder. Comprehensive Psychiatry, 58, 165-171. doi: 10.1016/j.comppsych.2014.11.018

Jazaieri, H., Goldin, P., Werner, K., Ziv, M., \& Gross, J. (2012). A randomized trial of MBSR versus aerobicexercise for social anxiety disorder. Journal of Clinical Psychology, 68, $715-731$

Jovanovic, V. (2016). The validity of the Satisfaction with Life Scale in adolescents and a comparison with single-item life satisfaction measures: a preliminary study. Quality of Life Research, 25, 3173-3180. doi: 10.1007/s11136-016-1331-5

Kabat-Zinn, J. (2000). Indra's net at work: The mainstreaming of Dharma practice in society. In G. Watson \& S. Batchelor (Eds.), The psychology of awakening: Buddhism, science, and our day-to-day lives (pp. 225-249). North Beach, ME: Weiser.

Kabat-Zinn, J. (2003). Mindfulness-based interventions in context: Past, present, and future. Clinical Psychology: Science and Practice, 10, 144-156.

Kashdan, T. B., Goodman, F. R., Machell, K. A., Kleiman, E. M., Monfort, S. S., Ciarrochi, J., \& Nezlek, J. B. (2014). A Contextual approach to experiential avoidance and social anxiety: Evidence From an experimental interaction and daily interactions of people with social 
anxiety disorder. Emotion, 14, 769-781.

Keng, S.-L., Smoski, M. J., Robins, C. J. (2011). Effects of mindfulness on psychological health: A review of empirical studies. Clinical Psychology Review, 31, 1041-1056. doi: 10.1016/j.cpr.2011.04.006

Khoury, B., Lecomte, T., Fortin, G., Masse, M., Therien, P., Bouchard, V., ... \& Hofmann, S. G. Mindfulness-based therapy: A comprehensive meta-analysis. Clinical Psychology Review, 33, 763-771.

Khoury, B., Sharma, M., Rush, S. E., \& Fournier, C. (2015). Mindfulness-based stress reduction for healthy individuals: A meta-analysis. Journal of Psychosomatic Research, 78, 519528.

Kircanski, K., Lieberman, M. D., \& Craske, M. G. (2012). Feelings into words: Contributions of language to exposure therapy. Psychological Science, 23, 1086-1091.

Kocovski, N. L., Fleming, J. E., \& Rector, N. A. (2009). Mindfulness and acceptance-based group therapy for social anxiety disorder: An open trial. Cognitive and Behavioral Practice, 16, 276-289. doi: 10.1016/j.cbpra.2008.12.004

Koszycki, D., Benger, M., Shlik, J., \& Bradwejn, J. (2007). Randomized trial of a meditationbased stress reduction program and cognitive behavior therapy in generalized social anxiety disorder. Behaviour Research and Therapy, 45, 2518-2526. doi: 10.1016/j.brat.2007.04.011

Koszycki, D., Thake, J., Mavounza, C., Daoust, J.-P., Taljaard, M., \& Bradwejn, J. (2016). Preliminary investigation of a mindfulness-based intervention for social anxiety disorder that integrates compassion meditation and mindful exposure. The Journal of Alternative and Complementary Medicine, 22, 363-374. doi: 10.1089/acm.2015.0108

LeDoux, J. (1998). Fear and the brain: where have we been, and where are we going? Biological Psychiatry, 44, 1229-1238.

Lei, M., Alden, L. E., Taylor, C. T. (2011). Differential effects of safety behaviour subtypes in social anxiety disorder. Behaviour Research and Therapy, 49, 665-675. doi: 10.1016/j.brat.2011.07.005

Lieberman, M. D., Eisenberger, N. I., Crockett, M. J., Tom, S. M., Pfeifer, J. H., \& Way, B. M. 
(2007). Putting feelings into words: affect labeling disrupts amygdala activity in response to affective stimuli. Psychological Science, 18, 421-428.

Lieberman, M. D., Inagaki, T. K., Tabibnia, G., \& Crockett, M. J. (2011). Subjective responses to emotional stimuli during labeling, reappraisal, and distraction. Emotion, 11, 468-480.

Liebowitz, M. R. (1987). Social phobia. Modern Problems of Pharmacopsychiatry, 22, 141-173. Lilja, J.L., Frodi-Lundgren, A., Johansson Hanse, J., Josefsson, T., Lundh, L.G., Sköld, C., Hansen, E.\& Broberg, A.G. (2011). Five Facets Mindfulness Questionnaire-Reliability and factor structure: A Swedish version. Cognitive Behaviour Therapy, 1-13.

Longe, O., Maratos, F. A., Gilbert, P., Evans, G., Volker, F., Rockliff, H., \& Rippon, G. (2010). Having a word with yourself: Neural correlates of self-criticism and self-reassurance. NeuroImage, 49, 1849-1856.

López, A., Sanderman, R., \& Schroevers, M. (2016). Mindfulness and self-compassion as unique and common predictors of affect in the general population. Mindfulness, 7, 1289-1296.

Lutz, A., Brefczynski-Lewis, J., Johnstone, T., \& Davidson, R. J. (2008). Regulation of the neural circuitry of emotion by compassion meditation: Effects of meditative expertise (neural effects of compassion). PLoS One, 3, e.1897.

MacBeth, A., \& Gumley, A. (2012). Exploring compassion: A meta-analysis of the association between self-compassion and psychopathology. Clinical Psychology Review, 32, 545552.

Michalak, J., Heidenreich, T., Meibert, P., \& Schulte, D. (2008). Mindfulness predicts relapse/recurrence in major depressive disorder after mindfulness-based cognitive therapy. Journal of Nervous and Mental Disease, 196, 630-633.

Montgomery, S. A., \& Asberg, M. (1979). A new depression scale designed to be sensitive to change. British Journal of Psychiatry, 134, 382-89. doi: 10.1192/bjp.134.4.382.

Mulkens, S., Bogels, S., de Jong, P., \& Louwers, J. (2001). Fear of blushing: Effects of task concentration training versus exposure in vivo on fear and psychology. Journal of Anxiety Disorders, 15, 413-432. 
Neely, M. E., Schallert, D. L., Mohammed, S. S., Roberts, R. M., \& Chen, Y.-J. (2009). Selfkindness when facing stress: The role of self-compassion, goal regulation, and support in college students' well-being. Motivation and Emotion, 33, 88 -97. doi:10.1007/s11031008-9119-8

Neff, K. D. (2003). Development and validation of a scale to measure self-compassion. Self and Identity, 2, 223-250.

Neff, K. D. (2016). The Self-Compassion Scale is a valid and theoretically coherent measure of self-compassion. Mindfulness, 7, 264-274. doi: 10.1007/s12671-015-0479-3

Neff, K. D. \& Dahm, K. A. (2014). Self-Compassion: What it is, what it does, and how it relates to mindfulness. In M. Robinson, B. Meier \& B. Ostafin (Eds.), Mindfulness and SelfRegulation (pp. 121-140). New York: Springer.

Neff, K. D. \& Germer, C. K. (2013). A pilot study and randomized controlled trial of the Mindful Self-Compassion program. Journal of Clinical Psychology, 69(1), 28-44.

Niles, A. N., Craske, M. G., Lieberman, M. D., \& Hur, C. (2015). Affect labeling enhances exposure effectiveness for public speaking anxiety. Behaviour Research and Therapy, 68, 27-36.

Norton, A. R., Abbott, M. J., Norberg, M. M., \& Hunt, C. (2014). A systematic review of mindfulness and acceptance-based treatments for social anxiety disorder. Journal of Clinical Psychology, 71, 283-301. doi: 10.1002/jclp.22144

Parsons, E., Luebbe, A., \& Clerkin, E. (2017). Testing the relationship between social anxiety schemas, mindfulness facets, and state and trait social anxiety symptoms. Mindfulness, 8 , 1634-1643.

Pavot W., \& Diener E. (1993). The affective and cognitive contest of self reports measures of subjective well-being. Social Indicators Research, 28, 1-20.

Piccirillo, M. L., Taylor Dryman, M., \& Heimberg, R. G. (2016). Safety behaviors in adults with social anxiety: Review and future directions. Behavior Therapy, 47, 675-687. doi: 10.1016/j.beth.2015.11.005

Piet, J., Hougaard, E., Hecksher, M. S., \& Rosenberg, N. K. (2010). A randomized pilot study of 
mindfulness-based cognitive therapy and group cognitive-behavioral therapy for young adults with social phobia. Scandinavian Journal of Psychology, 51, 403-410. doi: 10.1111/j.1467-9450.2009.00801.x

Preacher, K. J., \& Hayes, A. F. (2008). Asymptotic and resampling strategies for assessing and comparing indirect effects in multiple mediator models. Behavior Research Methods, 40, 879-891.

Raes, F., Pommier, E., Neff, K. D., \& Van Gucht, D. (2011). Construction and factorial validation of a short form of the Self-Compassion Scale. Clinical Psychology \& Psychotherapy, 18, 250-255.

Ramel, W., Goldin, P. R., Carmona, P. E., \& McQuaid, J. R. (2004). The effects of mindfulness meditation on cognitive processes and affect in patients with past depression. Cognitive Therapy and Research, 28, 433-455.

Rapee, R. M., \& Heimberg, R. G. (1997). A cognitive-behavioral model of anxiety in social phobia. Behaviour Research and Therapy, 35, 741-756. doi: 10.1016/S00057967(97)00022-3

Rapee, R. M., \& Lim, L. (1992). Discrepancy between self and observer ratings of performance in social phobics. Journal of Abnormal Psychology, 101, 727-731.

Reibel, D. K., Greeson, J. M., Brainard, G. C., \& Rosenweig, S. (2001). Mindfulness-based stress reduction and health-related quality of life in a heterogeneous patient population. General Hospital Psychiatry, 23, 183-192.

Robins, C. J., Keng, S. L., Ekblad, A. G., \& Brantley, J. G. (2012). Effects of mindfulness-based stress reduction on emotional experience and expression: a randomized controlled trial. Journal of Clinical Psychology, 68, 117-31.

Rodebaugh, T. L., Holaway, R. M., \& Heimberg, R. G. (2004). The treatment of social anxiety disorder. Clinical Psychology Review, 24, 883-908.

Rohleder, N., Chen, E., Wolf, J. M., \& Miller, G. E. (2008). The psychobiology of trait shame in young women: extending the social self-preservation theory. Health Psychology, 27, 523.

Rosenberg, M. (1965). Society and the adolescent self-image. Princeton, NJ: Princeton 
University Press.

Royuela-Colomer, E., \& Calvete, E. (2016). Mindfulness facets and depression in adolescents: rumination as a mediator. Mindfulness, 7, 1092-1102.

Sbarra, D. A., Smith, H. L., \& Mehl, M. R. (2012). When leaving your ex, love yourself: Observational ratings of self-compassion predict the course of emotional recovery following marital separation. Psychological Science, 23, 261-269.

Schmitt, D. P., \& Allik, J. (2005). Simultaneous administration of the Rosenberg Self-Esteem Scale in 53 nations: Exploring the universal and culture-specific features of global selfesteem. Journal of Personality and Social Psychology, 89, 623-642.

Schneier, F. R., Heckelman, L. R., Garfinkel, R., Campeas, R., Fallon, B.A., Gitow, A.,... \& Liebowitz M. R. (1994). Functional impairment in social phobia. J Clin Psychiatry, 55, $322-331$.

Sinclair, S., Blais, M., Gansler, D., Sandberg, E., Bistis, K., \& LoCicero. (2010). Psychometric properties of the Rosenberg Self-Esteem Scale: Overall and across demographic groups living within the United States. Evaluation \& the Health Professions, 33, 56-80. doi: $10.1177 / 0163278709356187$

Sirois, F., \& Tosti, N. (2012). Lost in the moment? An investigation of procrastination, mindfulness, and well-being. Journal of Rational-Emotive \& Cognitive-Behavioral Therapy, 30, 237-248. doi: 10.1007/s10942-012-0151-y

Sprinkle, S. D., Lurie, D., Insko, S. L., Atkinson, G., Jones, G. L., Logan, A. R., \& Bissada, N. N. (2002). Criterion validity, severity cut scores, and test-retest reliability of the Beck Depression Inventory-II in a university counseling center sample. Journal of Counseling Psychology, 49, 381-385. doi: 10.1037/0022-0167.49.3.381

Statistics Canada. (2015). Health State Descriptions for Canadians, Part 3: Social phobia. Retrieved from http://www.statcan.gc.ca/pub/82-619-m/2012004/sections/sectionbeng.htm\#a3

Tabachnick, B. G. \& Fidell, L. S. (2001). Using Multivariate Statistics. $4^{\text {th }}$ ed. Boston, MA: Allyn and Bacon.

Torrisi, S. J., Lieberman, M. D., Bookheimer, S. Y., \& Altshuler, L. L. (2013). Advancing understanding of affect labeling with dynamic causal modeling. NeuroImage, 82, 481- 
488.

Van Dam, N. T., Sheppard, S. C., Forsyth, J. P., \& Earleywine, M. (2011). Self-compassion is a better predictor than mindfulness of symptom severity and quality of life in mixed anxiety and depression. Journal of Anxiety Disorders, 25, 123-130. doi: 10.1016/j.janxdis.2010.08.011

Vassilopoulos, S. (2008). Social anxiety and ruminative self-focus. Journal of Anxiety Disorders, 22, 8960-8967.

Vassilopoulos, S., \& Watkins, E. (2009). Adaptive and maladaptive self-focus: A pilot extension study with individuals high and low in fear of negative evaluation. Behavior Therapy, 40, 181-189.

Vøllestad, J., Nielsen, M. B., \& Nielsen, G. H. (2012). Mindfulness-and acceptance-based interventions for anxiety disorders: A systematic review and meta-analysis. British Journal of Clinical Psychology, 51, 239-260.

Weissman, M. M., \& Bothwell, S. (1976). Assessment of social adjustment by patient selfreport. Archives of General Psychiatry, 33, 1111-1115.

Weissman, M. M., Olfson, M., Gameroff, M. J., Feder, A., \& Fuentes, M. (2001). A comparison of three scales for assessing social functioning in primary care. American Journal of Psychiatry, 158, 460-466.

Werner, K. H., Jazaieri, H., Goldin, P. R., Ziva, M., Heimberg, R. G., \& Gross, J. J. (2012). Selfcompassion and social anxiety disorder. Anxiety, Stress, \& Coping, 25, 543-558. doi: 10.1080/10615806.2011.608842

Wittchen, H. U. \& Fehm, L. (2001). Epidemiology, patterns of comorbidity, and associated disabilities of social phobia. Psychiatric Clinics of North America, 24, 617-641. doi: 10.1016/S0193-953X(05)70254-9

Woodruff, S. C., Glass, C. R., Arnkoff, D. B., Crowley, K. J., Hindman, R. K., \& Hirschhorn, E. W. (2014). Comparing self-compassion, mindfulness, and psychological inflexibility as predictors of psychological health. Mindfulness, 5, 410-421.

Woods, H., \& Proeve, M. (2014). Relationships of mindfulness, self-compassion, and meditation experience with shame-proneness. Journal of Cognitive Psychotherapy, 28, 20-33. 


\section{Appendix A: Dominance Analysis Outputs}

Table A1 Dominance matrix for LSAS

\begin{tabular}{lcccccc}
\hline & SCS-SF & Observe & Nonreact & Describe & Awareness & Nonjudge \\
\hline SCS-SF & - & 1.00 & 1.00 & 1.00 & 1.00 & 1.00 \\
Observe & .00 & - & .25 & .00 & .31 & .25 \\
Nonreact & .00 & .75 & - & .00 & .56 & .44 \\
Describe & .00 & 1.00 & 1.00 & - & 1.00 & 1.00 \\
Awareness & .00 & .69 & .44 & .00 & - & .50 \\
Nonjudge & .00 & .75 & .56 & .00 & .50 & -
\end{tabular}

Table A2 Dominance matrix for SPIN

\begin{tabular}{lcccccc}
\hline & SCS-SF & Observe & Nonreact & Describe & Awareness & Nonjudge \\
\hline SCS-SF & - & 1.00 & 1.00 & 1.00 & 1.00 & 1.00 \\
Observe & .00 & - & .50 & .00 & .25 & .38 \\
Nonreact & .00 & .50 & - & .00 & .06 & .31 \\
Describe & .00 & 1.00 & 1.00 & - & .75 & 1.00 \\
Awareness & .00 & .75 & .94 & .25 & - & 1.00 \\
Nonjudge & .00 & .63 & .69 & .00 & .00 & -
\end{tabular}


Table A3 Dominance matrix for BDI-II

\begin{tabular}{lcccccc}
\hline & SCS-SF & Observe & Nonreact & Describe & Awareness & Nonjudge \\
\hline SCS-SF & - & 1.00 & 1.00 & 1.00 & 1.00 & 1.00 \\
Observe & .00 & - & .13 & .00 & .00 & .06 \\
Nonreact & .00 & .88 & - & .25 & .00 & .50 \\
Describe & .00 & 1.00 & .75 & - & .00 & .50 \\
Awareness & .00 & 1.00 & 1.00 & 1.00 & - & 1.00 \\
Nonjudge & .00 & .94 & .50 & .50 & .00 & -
\end{tabular}

Table A4 Dominance matrix for RSES

\begin{tabular}{lcccccc}
\hline & SCS-SF & Observe & Nonreact & Describe & Awareness & Nonjudge \\
\hline SCS-SF & - & 1.00 & 1.00 & 1.00 & 1.00 & 1.00 \\
Observe & .00 & - & .50 & .31 & .00 & .44 \\
Nonreact & .00 & .50 & - & .50 & .00 & .63 \\
Describe & .00 & .69 & .50 & - & .00 & .38 \\
Awareness & .00 & 1.00 & 1.00 & 1.00 & - & 1.00 \\
Nonjudge & .00 & .56 & .38 & .63 & .00 & -
\end{tabular}


Table A5 Dominance matrix for SAS-SR

\begin{tabular}{lcccccc}
\hline & SCS-SF & Observe & Nonreact & Describe & Awareness & Nonjudge \\
\hline SCS-SF & - & 1.00 & 1.00 & 1.00 & 1.00 & 1.00 \\
Observe & .00 & - & .31 & .00 & .00 & .38 \\
Nonreact & .00 & .69 & - & .38 & .00 & .75 \\
Describe & .00 & 1.00 & .63 & - & .00 & .69 \\
Awareness & .00 & 1.00 & 1.00 & 1.00 & - & 1.00 \\
Nonjudge & .00 & .63 & .25 & .31 & .00 & -
\end{tabular}

Table A6 Dominance matrix for SWLS

\begin{tabular}{lcccccc}
\hline & SCS-SF & Observe & Nonreact & Describe & Awareness & Nonjudge \\
\hline SCS-SF & - & 1.00 & 1.00 & 1.00 & 1.00 & 1.00 \\
Observe & .00 & - & .50 & .75 & .50 & .63 \\
Nonreact & .00 & .50 & - & .75 & .88 & 1.00 \\
Describe & .00 & .25 & .25 & - & .44 & .25 \\
Awareness & .00 & .50 & .13 & .56 & - & .63 \\
Nonjudge & .00 & .38 & .00 & .75 & .38 & -
\end{tabular}

